Research Article

\title{
The Nonsteady Boussinesq System with Mixed Boundary Conditions including Conditions of Friction Type
}

\author{
Tujin Kim \\ Institute of Mathematics, State Academy of Sciences, Pyongyang, Democratic People's Republic of Korea \\ Correspondence should be addressed to Tujin Kim; math.tujin@star-co.net.kp
}

Received 6 March 2020; Accepted 25 May 2020; Published 3 September 2020

Academic Editor: Yoshikazu Giga

Copyright (C) 2020 Tujin Kim. This is an open access article distributed under the Creative Commons Attribution License, which permits unrestricted use, distribution, and reproduction in any medium, provided the original work is properly cited.

In this paper, we are concerned with the nonsteady Boussinesq system under mixed boundary conditions. The boundary conditions for fluid may include Tresca slip, leak and one-sided leak conditions, velocity, static (or total) pressure, rotation, and stress (or total stress) together, and the boundary conditions for temperature may include Dirichlet, Neumann, and Robin conditions together. Relying on the relations among strain, rotation, normal derivative of velocity, and shape of the boundary surface, we get variational formulation. The formulations consist of a variational inequality for velocity due to the boundary conditions of friction type and a variational equation for temperature. For the case of boundary conditions including the static pressure and stress, we prove that if the data of the problem are small enough and compatibility conditions at the initial instance are satisfied, then there exists a unique solution on the given interval. For the case of boundary conditions including the total pressure and total stress, we prove the existence of a solution without restriction on the data and parameters of the problem.

\section{Introduction}

In this paper, we are concerned with the Boussinesq equation for heat convection

$$
\left\{\begin{array}{l}
\frac{\partial v}{\partial t}-2 \nabla \cdot(\mu(\theta) \mathscr{E}(v))+(v \cdot \nabla) v+\nabla p=\left(1-\alpha_{0} \theta\right) f \\
\nabla \cdot v=0 \\
\frac{\partial \theta}{\partial t}-\nabla \cdot(\kappa(\theta) \nabla \theta)+v \cdot \nabla \theta=g \\
v(0)=v_{0}, \theta(0)=\theta_{0}
\end{array}\right.
$$

under mixed boundary conditions. Here, $v, p$, and $\theta$ are, respectively, velocity, pressure, and temperature, and $\alpha_{0}$ is the parameter for buoyancy effect, $f$ is the body force, $g$ is the heat source, $\mu$ is the viscosity, and $\kappa$ is the thermal conductivity. The strain tensor $\mathscr{E}(v)$ is the one with the components $\varepsilon_{i j}(v)=(1 / 2)\left(\partial_{x_{i}} v_{j}+\partial_{x_{j}} v_{i}\right)$. System (1) is a special case of

$$
\left\{\begin{array}{l}
\frac{\partial v}{\partial t}-2 \nabla \cdot(\mu(\theta) \mathscr{E}(v))+(v \cdot \nabla) v+\nabla p=\left(1-\alpha_{0} \theta\right) f \\
\nabla \cdot v=0 \\
\frac{\partial \theta}{\partial t}-\nabla \cdot(\kappa(\theta) \nabla \theta)+v \cdot \nabla \theta-\alpha_{2} \mu(\theta) \mathscr{E}(v): \mathscr{E}(v)=\alpha_{1} \theta f \cdot v+g, \\
v(0)=v_{0}, \theta(0)=\theta_{0},
\end{array}\right.
$$

which is a mathematical model for nonsteady motion of heat-conducting incompressible Newtonian fluid. Here, $\alpha_{1}$ is the parameter for dissipation of energy due to expansion, $\alpha_{2}$ is a positive real number, and for two matrices $A=\left\{a_{i j}\right\}$ and $B=\left\{b_{i j}\right\}, A: B=\sum_{i j} a_{i j} b_{i j}$ and $|A|=\left(\sum_{i j} a_{i j}^{2}\right)^{1 / 2}$. The term $\mu(\theta) \mathscr{E}(v): \mathscr{E}(v)$ represents the dissipation of energy due to viscosity (the Joule effect). Owing to the dissipation of energy due to viscosity $\mu(\theta) \mathscr{E}(v): \mathscr{E}(v)$, study of (2) is usually more difficult than the Boussinesq system.

For the papers concerned with (2), we refer to Introduction of [1]. Here, we more mention [2-5] concerned with (2), where $\alpha_{1}=0$. In [2], the problem under nonhomogeneous Dirichlet 
boundary conditions for velocity and temperature in the timedependent domain was studied, and existence of a local-in-time solution or existence of the solution on the given interval for small data was proved. In [3], existence of a strong solution and periodic solution for the $2 \mathrm{D}$ problem was studied under the boundary conditions and domain as above. In [4], under homogeneous Dirichlet boundary conditions for velocity and temperature, existence of a strong solution and periodic solution were studied when data of the problem are small enough. Łukaszewicz and Krzyżanowski [5] dealt with the initial boundary value problem on a time-dependent domain with the homogeneous Dirichlet boundary condition for velocity and temperature, and they proved the existence and uniqueness of local weak solutions and the existence of a global weak solution for small initial data.

Several papers are concerned with (1). In $[6,7]$, the existence and uniqueness (for 2D) of a solution to the problem were studied under the homogeneous Dirichlet boundary condition for velocity and mixture of nonhomogeneous Dirichlet and Neumann boundary conditions for temperature. In [8], for the problem with nonhomogeneous Dirichlet boundary conditions for velocity and temperature, the existence of the time periodic solution was proved (see [9]). In [10-13], problem (1) on the time-dependent domain was studied under the nonhomogeneous Dirichlet boundary condition for velocity and temperature. In $[14,15]$, the problem on exterior domains with the homogeneous Dirichlet boundary condition for velocity and nonhomogeneous Dirichlet boundary condition for temperature was studied. In [16], problem (1) was studied under the mixture of the nonhomogeneous Dirichlet boundary condition and the stress boundary condition for fluid and the mixture of nonhomogeneous Dirichlet, Neumann, and Robin boundary conditions for temperature. They proved the existence of a unique local-in-time solution under a compatibility condition at the initial instance (see (27) and (31) of [16]). In [17], problem (1) in the cylindrical pipe with inflow and outflow was studied under slip boundary conditions for velocity and the Neumann conditions for temperature. In that, it was proved that there exists a solution on the given interval when norms of derivatives in the direction along the cylinder of the initial velocity, initial temperature, and the external force are small enough. In [18], the existence of a solution to problem (1) on the time-dependent domain was studied under the mixture of the Dirichlet condition of velocity, total pressure, and rotation boundary conditions for fluid and the mixture of Dirichlet, Neumann, and Robin boundary conditions for temperature.

On the contrary, for movement of fluid $(v, p)$, different kinds of boundary conditions are used, and in practice, we deal with the mixture of some kinds of boundary conditions. On some portions of the boundary, we can use boundary conditions with stress or rotation, whereas when there is flux through a portion of the boundary, we can deal with the static pressure $p$ or the total pressure (Bernoulli's pressure) $(1 / 2)|v|^{2}+p$ boundary conditions. There are many literature studies for the Navier-Stokes problem with mixed boundary conditions (see Introduction of $[19,20]$ and references therein). Recently, Navier-Stokes system with mixed boundary conditions including friction-type conditions was studied (cf. [20, 21]).

In [1], problem (2) is studied under mixed boundary conditions, and the boundary conditions for fluid may include
Tresca slip, leak and one-sided leak conditions, velocity, total pressure, rotation, and total stress together, and the conditions for temperature may include Dirichlet, Neumann, and Robin conditions together. From the result of [1], we can get results for (1) with the boundary conditions as in [1]; however, the result demands that the parameter for buoyancy effect $\alpha_{0}$ is small enough in accordance with the data of the problem, and the solution includes "defect measure" as in [22]. Also, for (2) and (1), the problem with a mixed boundary condition including the static pressure (not total pressure) and stress (not total stress) together is not yet considered.

When one of static pressure, stress, or the outflow boundary condition is given on a portion of the boundary, for the initial boundary value problems of the Navier-Stokes equations, the existence of a unique local-in-time solution and a unique solution on a given interval for small data of the problem are proved. From the mathematical point of view, the main difficulty of such problems results from the fact that in a priori estimation of solution, the term arising from the nonlinear term $(v \cdot \nabla) v$ is not canceled (cf. Preface in [23]).

In the present paper, we are first concerned with heat convection equation (1) under mixed boundary conditions including the static pressure and stress. The boundary conditions for fluid may include conditions of friction type (Tresca slip, threshold leak, and one-sided leak conditions), velocity, static pressure, rotation, and stress together, and the conditions for temperature may include Dirichlet, Neumann, and Robin conditions together. Due to the boundary conditions of friction type, it is difficult to follow the methods in $[16,20]$. The main difficulty of this problem is from the estimate of approximate solutions, and due to simultaneous velocity and temperature, the estimate is more difficult than the case of the Navier-Stokes equations. Also, in this paper, we prove the existence of a solution to (1) with the boundary conditions as in [1] without restriction on the parameter for buoyancy effect $\alpha_{0}$.

This paper consists of 5 sections. In the last part of Section 1, we give notations.

In Section 2, the problems to study and assumptions for future are stated. According to the boundary conditions for fluid, Problems I and II are distinguished. Problem I includes the static pressure and the stress conditions, whereas Problem II includes the total pressure and the total stress boundary conditions. Assumption for Problem I is stronger than the one for Problem II.

In Section 3, we first get a variational formulation for Problem I which consists of six formulae with six unknown functions, that is, using velocity, tangent stress on slip surface, normal stress on the leak surface, normal stresses on one-sided leak surfaces, and temperature together as unknown functions (Problem I-VE). Then, we get a new variational formulation for Problem I consisting of one variational inequality for velocity and a variational equation for temperature (Problem I-VI).

The variational formulation for Problem II is obtained in the same way as in [1], and smoothness of the solution with respect to $t$ is weaker than the one in Problem I. In the end of Section 3, the main results of this paper are stated (Theorems 1 and 2). The main result for Problem I asserts that if the data of the problem are small enough and compatibility conditions at 
the initial time (conditions 4 and 6 of Theorem 1) hold, then there exists a unique smooth solution. The main result for Problem II asserts the existence of a solution without restriction on the parameter for buoyancy unlike [1].

Section 4 is devoted to the proof of Theorem 1. To this end, first in Section 4.1, we consider an approximate problem, where the variational inequality for velocity is replaced by an equation with the gradient of the Moreau regularization of the functional due to the boundary conditions of friction type. Developing the method for the proof of Theorem 4.4 of [21], we get existence and estimations of approximate solutions for small data under the compatibility conditions at initial time. In Section 4.2, we complete the proof of the existence and uniqueness of a solution.

Section 5 is devoted to the proof of Theorem 2. The existence of solutions to an approximate problem and relative compactness of the set of solutions are studied. Then, passing to limit, we get the conclusion.

Throughout this paper, we will use the following notation. Let $\Omega$ be a connected bounded open subset of $\mathbb{R}^{l}, l=2,3 . \partial \Omega \in C^{0,1}$,

$$
\partial \Omega=\cup_{i=1}^{11} \bar{\Gamma}_{i}=\bar{\Gamma}_{D} \cup \bar{\Gamma}_{R},
$$

$\Gamma_{D} \cap \Gamma_{R}=\varnothing, \Gamma_{i} \cap \Gamma_{j}=\varnothing$ for $i \neq j$, and $\Gamma_{i}=\cup_{j} \Gamma_{i j}$, where $\Gamma_{i j}$ are connected open subsets of $\partial \Omega$, and $\Gamma_{i j} \in C^{2,1}$ for $i=$ $2,3,7$ and $\Gamma_{i j} \in C^{1}$ for others. When $X$ is a Banach space, $\mathbf{X}=X^{l}$ and $X^{*}$ is a dual space of $X$. Let $W^{\alpha, p}(\Omega)$ be Sobolev spaces; $H^{1}(\Omega)=W^{1,2}(\Omega)$, and so $\mathbf{H}^{1}(\Omega)=\left\{H^{1}(\Omega)\right\}^{l}$. An inner product and norm in the spaces $L^{2}(\Omega)$ and $L^{2}(\Omega)$ are denoted, respectively, by $(\cdot, \cdot)$ and $\|\cdot\|$, and $\langle\cdot, \cdot\rangle$ means the duality pairing between a Sobolev space $X$ and its dual one. Also, $(\cdot, \cdot)_{\Gamma_{i}}$ is an inner product in $\mathbf{L}^{2}\left(\Gamma_{i}\right)$ or $L^{2}\left(\Gamma_{i}\right)$, and $\langle\cdot, \cdot\rangle_{\Gamma_{i}}$ means the duality pairing between $\mathbf{H}^{1 / 2}\left(\Gamma_{i}\right)$ and $\mathbf{H}^{-(1 / 2)}\left(\Gamma_{i}\right)$ or between $H^{1 / 2}\left(\Gamma_{i}\right)$ and $H^{-(1 / 2)}\left(\Gamma_{i}\right)$. The inner product and norms in $\mathbb{R}^{l}$, respectively, are denoted by $(\cdot, \cdot)_{\mathbb{R}^{l}}$ and $|\cdot|$. Sometimes, the inner product between $a$ and $b$ in $\mathbb{R}^{l}$ is denoted by $a \cdot b$.

Let $n(x)$ and $\tau(x)$ be, respectively, outward normal and tangent unit vectors at $x$ in $\partial \Omega$. When $f \in H^{-1 / 2}\left(\Gamma_{i}\right)$, if $\langle f, w\rangle_{\Gamma_{i}} \geq 0(\leq 0) \forall w \in C_{0}^{\infty}\left(\Gamma_{i}\right)$ with $w \geq 0$, then we denote by $f \geq 0(\leq 0)$ on $\Gamma_{i}$. For convergence in spaces, $\longrightarrow, \rightarrow$, and $二$ mean, respectively, strong, weak, and weak * convergence. Derivative of $f(t, x)$ with respect to $t$ is denoted by $f^{\prime}$. We also assume that $0<T<\infty$.

\section{Problems and Assumptions}

For temperature, we are concerned with the boundary conditions

$$
\begin{aligned}
& \left.\theta\right|_{\Gamma_{D}}=0, \\
& \left.\left(\kappa(\theta) \frac{\partial \theta}{\partial n}+\beta(x) \theta\right)\right|_{\Gamma_{R}}=g_{R}(t, x), \\
& \quad \beta(x), g_{R}(t, x)-\text { given functions on } \Gamma_{R},(0, T) \times \Gamma_{R} .
\end{aligned}
$$

Stress tensor $S$ is the one with components $s_{i j}=-p \delta_{i j}+2 \mu \varepsilon_{i j}(v)$, and stress vector on the boundary surface is $\sigma(v, p)=S \cdot n$. The value of the normal stress vector on the boundary surface is $\sigma_{n}(v, p)=\sigma \cdot n$. And $\sigma_{\tau}(v, p)=\sigma(v, p)-\sigma_{n}(v, p) n$. Total stress tensor $S^{t}$ is the one with components $s_{i j}^{t}=-\left(p+(1 / 2)|v|^{2}\right) \delta_{i j}+2 \mu(\theta) \varepsilon_{i j}(v)$, and the total stress vector on the boundary surface is $\sigma^{t}(\theta, v, p)=S^{t} \cdot n$. The value of the total normal stress vector on the boundary surface is $\sigma_{n}^{t}(\theta, v, p)=\sigma^{t} \cdot n$. And $\sigma_{\tau}^{t}(\theta, v, p)=\sigma^{t}(\theta, v, p)-\sigma_{n}^{t}(\theta, v, p) n$.

For Problem I, we assume that $\mu$ and $\kappa$ are independent of $\theta$. Thus, Problem I is the one with the boundary conditions

$$
\begin{aligned}
& \left.v\right|_{\Gamma_{1}}=0, \\
& \left.v_{\tau}\right|_{\Gamma_{2}}=0,-\left.p\right|_{\Gamma_{2}}=\phi_{2}, \\
& \left.v_{n}\right|_{\Gamma_{3}}=0, \operatorname{rot} v \times\left. n\right|_{\Gamma_{3}}=\frac{\phi_{3}}{\mu}, \\
& \left.v_{\tau}\right|_{\Gamma_{4}}=0,\left.\left(-p+2 \mu \varepsilon_{n n}(v)\right)\right|_{\Gamma_{4}}=\phi_{4}, \\
& \left.v_{n}\right|_{\Gamma_{5}}=0,\left.2\left(\mu \varepsilon_{n \tau}(v)+\alpha v_{\tau}\right)\right|_{\Gamma_{5}}=\phi_{5}, \alpha: a \text { matrix, } \\
& \left.\left(-p n+2 \mu \varepsilon_{n}(v)\right)\right|_{\Gamma_{6}}=\phi_{6}, \\
& \left.v_{\tau}\right|_{\Gamma_{7}}=0,\left.\left(-p+\mu \frac{\partial v}{\partial n} \cdot n\right)\right|_{\Gamma_{7}}=\phi_{7}, \\
& v_{n}=0,\left|\sigma_{\tau}(v)\right| \leq g_{\tau}, \sigma_{\tau}(v) \cdot v_{\tau}+g_{\tau}\left|v_{\tau}\right|=0 \quad \text { on } \Gamma_{8}, \\
& v_{\tau}=0,\left|\sigma_{n}(v, p)\right| \leq g_{n}, \sigma_{n}(v, p) v_{n}+g_{n}\left|v_{n}\right|=0 \quad \text { on } \Gamma_{9}, \\
& v_{\tau}=0, v_{n} \geq 0, \sigma_{n}(v, p)+g_{+n} \geq 0,\left(\sigma_{n}(v, p)+g_{+n}\right) v_{n}=0 \quad \text { on } \Gamma_{10}, \\
& v_{\tau}=0, v_{n} \leq 0, \sigma_{n}(v, p)-g_{-n} \leq 0,\left(\sigma_{n}(v, p)-g_{-n}\right) v_{n}=0 \quad \text { on } \Gamma_{11},
\end{aligned}
$$


and Problem II is the one with the boundary conditions

$$
\begin{aligned}
& \left.v\right|_{\Gamma_{1}}=0 \\
& \left.v_{\tau}\right|_{\Gamma_{2}}=0,-\left.\left(p+\frac{1}{2}|v|^{2}\right)\right|_{\Gamma_{2}}=\phi_{2} \\
& \left.v_{n}\right|_{\Gamma_{3}}=0, \operatorname{rot} v \times\left. n\right|_{\Gamma_{3}}=\frac{\phi_{3}}{\mu(\theta)}, \\
& \left.v_{\tau}\right|_{\Gamma_{4}}=0,\left.\left(-p-\frac{1}{2}|v|^{2}+2 \mu(\theta) \varepsilon_{n n}(v)\right)\right|_{\Gamma_{4}}=\phi_{4}, \\
& \left.v_{n}\right|_{\Gamma_{5}}=0,\left.2\left(\mu(\theta) \varepsilon_{n \tau}(v)+\alpha v_{\tau}\right)\right|_{\Gamma_{5}}=\phi_{5}, \alpha \text { : a matrix, } \\
& \left.\left(-p n-\frac{1}{2}|v|^{2} n+2 \mu(\theta) \varepsilon_{n}(v)\right)\right|_{\Gamma_{6}}=\phi_{6}, \\
& \left.v_{\tau}\right|_{\Gamma_{7}}=0,\left.\left(-p-\frac{1}{2}|v|^{2}+\mu(\theta) \frac{\partial v}{\partial n} \cdot n\right)\right|_{\Gamma_{7}}=\phi_{7}, \\
& v_{n}=0,\left|\sigma_{\tau}^{t}(\theta, v)\right| \leq g_{\tau}, \sigma_{\tau}^{t}(\theta, v) \cdot v_{\tau}+g_{\tau}\left|v_{\tau}\right|=0 \quad \text { on } \Gamma_{8}, \\
& v_{\tau}=0,\left|\sigma_{n}^{t}(\theta, v, p)\right| \leq g_{n}, \sigma_{n}^{t}(\theta, v, p) v_{n}+g_{n}\left|v_{n}\right|=0 \quad \text { on } \Gamma_{9} \text {, } \\
& v_{\tau}=0, v_{n} \geq 0, \sigma_{n}^{t}(\theta, v, p)+g_{+n} \geq 0,\left(\sigma_{n}^{t}(\theta, v, p)+g_{+n}\right) v_{n}=0 \quad \text { on } \Gamma_{10} \text {, } \\
& v_{\tau}=0, v_{n} \leq 0, \sigma_{n}^{t}(\theta, v, p)-g_{-n} \leq 0,\left(\sigma_{n}^{t}(\theta, v, p)-g_{-n}\right) v_{n}=0 \quad \text { on } \Gamma_{11} \text {, }
\end{aligned}
$$

where $\varepsilon_{n}(v)=\mathscr{E}(v) n, \varepsilon_{n n}(v)=(\mathscr{E}(v) n, n)_{\mathbb{R}^{l}}, \varepsilon_{n \tau}(v)=\mathscr{E}(v)$ $n-\varepsilon_{n n}(v) n, \quad v_{\tau}=v-(v \cdot n) n, \quad v_{n}=v \cdot n, \quad$ and $h_{i}, \phi_{i}, \alpha_{j k}$ (components of matrix $\alpha$ ) are given functions or vectors of functions of $t, x$ on $\Sigma_{i}=(0, T) \times \Gamma_{i}$. For convenience in what follows, the problems with boundary conditions (5) and (6) are called, respectively, the case of static pressure and the case of total pressure.

Let

$$
\begin{aligned}
& \mathbf{V}=\left\{u \in \mathbf{H}^{1}(\Omega): \operatorname{div} u=0,\left.u\right|_{\Gamma_{1}}=0,\left.u_{\tau}\right|_{\left(\Gamma_{2} \cup \Gamma_{4} \cup \Gamma_{7} \cup \Gamma_{9} \cup \Gamma_{10} \cup \Gamma_{11}\right)}=0,\left.u_{n}\right|_{\left(\Gamma_{3} \cup \Gamma_{5} \cup \Gamma_{8}\right)}=0\right\}, \\
& H: \text { completion in } \mathbf{L}^{2}(\Omega) \text { of } \mathbf{V}, \\
& K(\Omega)=\left\{u \in \mathbf{V}:\left.u_{n}\right|_{\Gamma_{10}} \geq 0,\left.u_{n}\right|_{\Gamma_{11}} \leq 0\right\}, \\
& H_{K}: \text { closure in } \mathbf{L}^{2}(\Omega) \text { of } K(\Omega), \\
& K(Q)=\left\{u \in L^{2}(0, T ; \mathbf{V}): u^{\prime} \in L^{2}\left(0, T ; \mathbf{V}^{*}\right) ;\left.u_{n}\right|_{\Gamma_{10}} \geq 0,\left.u_{n}\right|_{\Gamma_{11}} \leq 0\right\}, \\
& W_{\Gamma_{D}}^{1,2}(\Omega)=\left\{y \in W^{1,2}(\Omega):\left.y\right|_{\Gamma_{D}}=0\right\} .
\end{aligned}
$$

We assume that $g_{\tau} \in L^{2}\left(\Gamma_{8}\right), g_{n} \in L^{2}\left(\Gamma_{9}\right), g_{+n} \in L^{2}\left(\Gamma_{10}\right)$, and that $g_{-n} \in L^{2}\left(\Gamma_{11}\right)$, and $g_{\tau}>0, g_{n}>0, g_{+n}>0$, and $g_{-n}>0$ for a.e. $x$ of the portions of boundary. Also, we use the following assumption.

Assumption 1 (for the case of static pressure). We assume the following:
(1) $\Gamma_{1} \neq \varnothing, \Gamma_{D} \neq \varnothing$, and

$$
\Gamma_{R} \subset\left(\cup_{i=1,3,5,8} \Gamma_{i}\right)
$$

(2) If $\Gamma_{i}$, where $i$ is 10 or 11 , is nonempty, then at least one of $\left\{\Gamma_{j}: j \in\{\{2,4,7,9-11\} \backslash\{i\}\}\right\}$ is nonempty, 
and there exists diffeomorphism in $C^{1}$ between $\Gamma_{i}$ and $\Gamma_{j}$.

(3) For the functions of (1),

$$
\begin{aligned}
& f \in W^{1, \infty}\left(0, T ; \mathbf{L}^{3}(\Omega)\right), \\
& g \in W^{1,2}\left(0, T ; W_{\Gamma_{D}}^{1,2}(\Omega)^{*}\right), \\
& \mu \text { and } \kappa \text { are independent of } \theta .
\end{aligned}
$$

(4) For the functions of (4) and (5),

$$
\begin{aligned}
& g_{R} \in W^{1,2}\left(0, T ; L^{4 / 3}\left(\Gamma_{R}\right)\right), \\
& \beta_{1} \geq \beta(x) \geq 0, \beta_{1} \text { - a constant, } \beta(x) \text { - measurable, } \\
& \phi_{i} \in W^{1, \infty}\left(0, T ; H^{-1 / 2}\left(\Gamma_{i}\right)\right), \quad i=2,4,7, \\
& \phi_{i} \in W^{1, \infty}\left(0, T ; \mathbf{H}^{-1 / 2}\left(\Gamma_{i}\right)\right), \quad i=3,5,6, \\
& \alpha_{i j} \in L^{\infty}\left(\Gamma_{5}\right) .
\end{aligned}
$$

Assumption 2 (for the case of total pressure). We assume (1) and (2) of Assumption 1 and the following:

$\left(3^{\prime}\right)$ For the functions of (1),

$$
\begin{aligned}
& f \in L^{\infty}\left(0, T ; \mathbf{L}^{3}(\Omega)\right), \\
& g \in L^{2}\left(0, T ; W_{\Gamma_{D}}^{1,2}(\Omega)^{*}\right), \\
& \mu \in C(\mathbb{R}), 0<\mu_{0} \leq \mu(\xi) \leq \mu_{1}<\infty, \quad \forall \xi \in \mathbb{R}, \\
& \kappa \in C(\mathbb{R}), 0<\kappa_{0} \leq \kappa(\xi) \leq \kappa_{1}<\infty, \quad \forall \xi \in \mathbb{R} .
\end{aligned}
$$

$$
\begin{aligned}
-2(\nabla \cdot(\mu \mathscr{E}(v)), u)= & 2(\mu \mathscr{E}(v), \mathscr{E}(u))-2(\mu \mathscr{E}(v) n, u)_{\cup_{i=2}^{11} \Gamma_{i}} \\
= & 2(\mu \mathscr{E}(v), \mathscr{E}(u))+2(\mu k(x) v, u)_{\Gamma_{2}}-(\mu \operatorname{rot} v \times n, u)_{\Gamma_{3}}+2(\mu S \widetilde{v}, \widetilde{u})_{\Gamma_{3}}-2\left(\mu \varepsilon_{n n}(v), u_{n}\right)_{\Gamma_{4}} \\
= & -2\left(\mu \varepsilon_{n \tau}(v), u\right)_{\Gamma_{5}}-2\left(\mu \varepsilon_{n}(v), u\right)_{\Gamma_{6}}-\left(\mu \frac{\partial v}{\partial n}, u\right)_{\Gamma_{7}}+(\mu k(x) v, u)_{\Gamma_{7}} \\
& -2\left(\mu \varepsilon_{n \tau}(v), u\right)_{\Gamma_{8}}-2\left(\mu \varepsilon_{n n}(v), u_{n}\right)_{\Gamma_{9}}-2\left(\mu \varepsilon_{n n}(v), u_{n}\right)_{\Gamma_{10}}-2\left(\mu \varepsilon_{n n}(v), u_{n}\right)_{\Gamma_{11}}
\end{aligned}
$$

where $S$ is the shape operator of the boundary surface (cf. (A.1) in [19]), $\widetilde{v}, \widetilde{u}$ are expressions of $v, u$ in a local coordinate system on $\Gamma_{3}$, and $\left.k(x)\right|_{\Gamma_{i}}=2 \times$ mean curvature of $\Gamma_{i}$.

For $p \in H^{1}(\Omega)$ and $u \in \mathbf{V}$, we have
3.1. Variational Formulations: The Case of Static Pressure. By Theorems 2.1 and 2.2 of [19], for $v \in \mathbf{H}^{2}(\Omega) \cap \mathbf{V}$ and $u \in \mathbf{V}$, we have

$$
\begin{aligned}
(\nabla p, u) & =-(p, \operatorname{div} u)+\left(p, u_{n}\right)_{\cup_{i=2}^{11} \Gamma_{i}} \\
& =\left(p, u_{n}\right)_{\Gamma_{2} \cup \Gamma_{4} \cup \Gamma_{7} \cup \Gamma_{9} \cup \Gamma_{10} \cup \Gamma_{11}}+(p n, u)_{\Gamma_{6}},
\end{aligned}
$$

\section{Variational Formulations and Main Results}

Since $\Gamma_{1} \neq \varnothing$ and $\Gamma_{D} \neq \varnothing$, by the Korn inequality and Poincaré inequality, we use

$$
(v, u)_{V}=(\mathscr{E}(v), \mathscr{E}(u)), \quad(y, z)_{W_{\Gamma_{D}}^{1,2}(\Omega)}=(\nabla y, \nabla z) .
$$

$$
\begin{aligned}
& g_{R} \in L^{2}\left(0, T ; L^{4 / 3}\left(\Gamma_{R}\right)\right), \\
& \beta_{1} \geq \beta(x) \geq 0, \beta_{1}-\text { a constant, } \beta(x) \text { - measurable, } \\
& \phi_{i} \in L^{2}\left(0, T ; H^{-(1 / 2)}\left(\Gamma_{i}\right)\right), \quad i=2,4,7, \\
& \phi_{i} \in L^{2}\left(0, T ; H^{-(1 / 2)}\left(\Gamma_{i}\right)\right), \quad i=3,5,6, \\
& \alpha_{i j} \in L^{\infty}\left(\Gamma_{5}\right) . \\
& \text { Remark } 1 . \text { On } \Gamma_{10}\left(\Gamma_{11}\right) \text {, only outflow (inflow) is possible, } \\
& \text { and so (2) of Assumption } 1 \text { is used to guarantee div } v=0 \text {. In } \\
& \text { Theorems 3.3 and 3.5 of [24], for the proof of equivalence of } \\
& \text { variational formulations to variational inequalities, this } \\
& \text { assumption was used via Lemma } 3.2 \text { of [24]. In this paper, } \\
& \text { this assumption is also necessary to guarantee equivalence }
\end{aligned}
$$$$
g_{R} \in L^{2}\left(0, T ; L^{4 / 3}\left(\Gamma_{R}\right)\right)
$$ 


$$
\begin{aligned}
(-\nabla \cdot(\kappa \nabla \theta), \varphi) & =(\kappa \nabla \theta, \nabla \varphi)-\left(\kappa \frac{\partial \theta}{\partial n}, \varphi\right)_{\Gamma_{R}} \\
& =(\kappa \nabla \theta, \nabla \varphi)+\left(\beta \theta-g_{R}, \varphi\right)_{\Gamma_{R}} .
\end{aligned}
$$

Taking into account (8) and $\left.v_{n}\right|_{\Gamma_{3} \cup \Gamma_{5} \cup \Gamma_{8}}=0$, for $v \in \mathbf{V}$, $\theta \in H^{1}(\Omega)$, and $\varphi \in W_{\Gamma_{D}}^{1,2}(\Omega)$, we have

$$
(v \cdot \nabla \theta, \varphi)=\left(v_{n} \theta, \varphi\right)_{\Gamma_{R}}-(\theta v, \nabla \varphi)=-(\theta v, \nabla \varphi) .
$$

By (14)-(17), we can see that smooth solutions $(v, p, \theta)$ of problems (1), (4), and (5) satisfy

$$
\left\{\begin{array}{l}
\left(\frac{\partial v}{\partial t}, u\right)+2(\mu \mathscr{E}(v), \mathscr{E}(u))+\langle(v \cdot \nabla) v, u\rangle+2(\mu k(x) v, u)_{\Gamma_{2}}+2(\mu S \widetilde{v}, \widetilde{u})_{\Gamma_{3}}+2(\alpha(x) v, u)_{\Gamma_{5}} \\
+(\mu k(x) v, u)_{\Gamma_{7}}-2\left(\mu \varepsilon_{n \tau}(v), u\right)_{\Gamma_{8}}+\left(p-2 \mu \varepsilon_{n n}(v), u_{n}\right)_{\Gamma_{9} \cup \Gamma_{10} \cup \Gamma_{11}} \\
=\left\langle\left(1-\alpha_{0} \theta\right) f, u\right\rangle+\sum_{i=2,4,7}\left\langle\phi_{i}, u_{n}\right\rangle_{\Gamma_{i}}+\sum_{i=3,5,6}\left\langle\phi_{i}, u\right\rangle_{\Gamma_{i}}, \quad \forall u \in \mathbf{V}, \\
\left(\frac{\partial \theta}{\partial t}, \varphi\right)+(\kappa \nabla \theta, \nabla \varphi)-(\theta v, \nabla \varphi)+(\beta \theta, \varphi)_{\Gamma_{R}}=\left(g_{R}, \varphi\right)_{\Gamma_{R}}+\langle g, \varphi\rangle, \quad \forall \varphi \in W_{\Gamma_{D}}^{1,2}(\Omega), \\
\left|\sigma_{\tau}(\theta, v)\right| \leq g_{\tau}, \sigma_{\tau}(\theta, v) \cdot v_{\tau}+g_{\tau}\left|v_{\tau}\right|=0, \quad \text { on } \Gamma_{8}, \\
\left|\sigma_{n}(v, p)\right| \leq g_{n}, \sigma_{n}(v, p) v_{n}+g_{n}\left|v_{n}\right|=0, \quad \text { on } \Gamma_{9}, \\
\left.\theta\right|_{\Gamma_{D}}=0 . \\
\sigma_{n}(v, p)+g_{+n} \geq 0,\left(\sigma_{n}(v, p)+g_{+n}\right) v_{n}=0, \quad \text { on } \Gamma_{10}, \\
\sigma_{n}(v, p)-g_{-n} \leq 0,\left(\sigma_{n}(v, p)-g_{-n}\right) v_{n}=0, \quad \text { on } \Gamma_{11},
\end{array}\right.
$$

Define $a_{01}(\cdot, \cdot), a_{11}(\cdot, \cdot, \cdot)$, and $f_{1}(t) \in V^{*}$ by

$$
\begin{aligned}
& a_{01}(w, u)= 2(\mu \mathscr{E}(w), \mathscr{E}(u))+2(\mu k(x) w, u)_{\Gamma_{2}} \\
&+2(\mu S \widetilde{w}, \widetilde{u})_{\Gamma_{3}}+2(\alpha(x) w, u)_{\Gamma_{5}}+(\mu k(x) w, u)_{\Gamma_{7}}, \\
& \forall w, u \in \mathbf{V},
\end{aligned}
$$

$a_{11}(v, u, w)=\langle(v \cdot \nabla) u, w\rangle, \quad \forall v, u, w \in \mathbf{V}$,$$
\left\langle f_{1}(t), u\right\rangle=\sum_{i=2,4,7}\left\langle\phi_{i}(t), u_{n}\right\rangle_{\Gamma_{i}}+\sum_{i=3,5,6}\left\langle\phi_{i}(t), u\right\rangle_{\Gamma_{i}}, \forall u \in \mathbf{V} .
$$

Define $b_{1}(\cdot, \cdot)$ and $g_{1}(t) \in\left(W_{\Gamma_{D}}^{1,2}(\Omega)\right)^{*}$ by

$$
\begin{aligned}
b_{1}(\theta, \varphi) & =(\kappa \nabla \theta, \nabla \varphi)+(\beta(x) \theta, \varphi)_{\Gamma_{R}}, \quad \forall \theta \in W^{1,2}(\Omega), \varphi \in W_{\Gamma_{D}}^{1,2}(\Omega), \\
\left\langle g_{1}(t), \varphi\right\rangle & =\left(g_{R}(t), \varphi\right)_{\Gamma_{R}}+\langle g(t), \varphi\rangle, \quad \forall \varphi \in W_{\Gamma_{D}}^{1,2}(\Omega) .
\end{aligned}
$$

Remark 2. Under (4) of Assumption 1, the duality product $\left\langle f_{1}, u\right\rangle$ of (19) has a meaning (cf. Remark 3.1 in [24]). By (9) and (10),

$$
\begin{aligned}
& f_{1} \in W^{1, \infty}\left(0, T ; \mathbf{V}^{*}\right), \\
& g_{1} \in W^{1,2}\left(0, T ; W_{\Gamma_{D}}^{1,2}(\Omega)^{*}\right) .
\end{aligned}
$$

Then, taking into account

$$
\begin{aligned}
\sigma_{\tau}(\theta, v) & =2 \mu \varepsilon_{n \tau}(v), \\
\sigma_{n}(\theta, v, p) & =-p+2 \mu \varepsilon_{n n}(v),
\end{aligned}
$$

and (18), we introduce the following variational formulation for problems (1), (4), and (5).

Problem (I-VE). Find $v \in K(Q), \theta \in L^{2}\left(0, T ; W_{\Gamma_{D}}^{1,2}(\Omega)\right)$, and $\left(\sigma_{\tau}, \sigma_{n}, \sigma_{+n}, \sigma_{-n}\right) \in L_{\tau}^{2}\left(\Gamma_{8}\right) \times L^{2}\left(\Gamma_{9}\right) \times H^{-(1 / 2)}\left(\Gamma_{10}\right) \times$ $H^{-(1 / 2)}\left(\Gamma_{11}\right)$ a.e. $t \in(0, T)$ such that $v(0)=v_{0}, \theta(0)=\theta_{0}$, and 


$$
\left\{\begin{array}{l}
\left\langle\frac{\partial v}{\partial t}, u\right\rangle+a_{01}(v, u)+a_{11}(v, v, u)-\left(\sigma_{\tau}, u_{\tau}\right)_{\Gamma_{8}}-\left(\sigma_{n}, u_{n}\right)_{\Gamma_{9}} \\
-\left\langle\sigma_{+n}, u_{n}\right\rangle_{\Gamma_{10}}-\left\langle\sigma_{-n}, u_{n}\right\rangle_{\Gamma_{11}}-\left\langle f-\alpha_{0} \theta f, u\right\rangle=\left\langle f_{1}, u\right\rangle, \quad \forall u \in L^{2}(0, T ; \mathbf{V}), \\
\left\langle\frac{\partial \theta}{\partial t}, \varphi\right\rangle+b_{1}(\theta, \varphi)-\langle\theta v, \nabla \varphi\rangle=\left\langle g_{1}, \varphi\right\rangle, \quad \forall \varphi \in L^{2}\left(0, T ; W_{\Gamma_{D}}^{1,2}(\Omega)\right), \\
\left|\sigma_{\tau}\right| \leq g_{\tau}, \sigma_{\tau} \cdot v_{\tau}+g_{\tau}\left|v_{\tau}\right|=0, \quad \text { on } \Gamma_{8}, \\
\left|\sigma_{n}\right| \leq g_{n}, \sigma_{n} v_{n}+g_{n}\left|v_{n}\right|=0, \quad \text { on } \Gamma_{9}, \\
\sigma_{+n}+g_{+n} \geq 0,\left\langle\sigma_{+n}+g_{+n}, v_{n}\right\rangle_{\Gamma_{10}}=0 \quad \text { on } \Gamma_{10}, \\
\sigma_{-n}-g_{-n} \leq 0,\left\langle\sigma_{-n}-g_{-n}, v_{n}\right\rangle_{\Gamma_{11}}=0, \quad \text { on } \Gamma_{11},
\end{array}\right.
$$

where $\mathbf{L}_{\tau}^{2}\left(\Gamma_{8}\right)$ is the subspace of $L^{2}\left(\Gamma_{8}\right)$ consisting of functions such that $(u, n)_{\mathbf{L}^{2}\left(\Gamma_{8}\right)}=0$.

Remark 3. Under Assumption 1, if a solution is smooth enough, $\quad\left(v \in L^{2}\left(0, T ; \mathbf{H}^{2}(\Omega)\right), \quad v^{\prime} \in L^{2}\left(0, T ; \mathbf{L}^{2}(\Omega)\right)\right.$, $\left.\theta \in L^{2}\left(0, T ; W^{2,2}(\Omega)\right), \theta^{\prime} \in L^{2}\left(0, T ; L^{2}(\Omega)\right)\right)$, then Problem $\mathrm{I}-\mathrm{VE}$ is equivalent to problems (1), (4), and (5) in the following sense.

By Theorem 3.4 of [24], at a.e., there exists $p(t)$ satisfying the first equation of $(1)$, and $(v, p)$ satisfies boundary condition (5). As given in Section 1, ch. 2 of [25], it is proved that $\theta$ satisfies the third equation of (1) and boundary condition (4).

We will find another variational formulation consisting of a variational inequality and a variational equation, which is equivalent to Problem I-VE if the solution is smooth enough (cf. Remark 4).

$$
\left\{\begin{array}{l}
\left\langle\frac{\partial v}{\partial t}, u\right\rangle+a_{01}(v, u)+a_{11}(v, v, u)-\left(\sigma_{\tau}, u_{\tau}\right)_{\Gamma_{8}}-\left(\sigma_{n}, u_{n}\right)_{\Gamma_{9}}-\left\langle\sigma_{+n}, u_{n}\right\rangle_{\Gamma_{10}} \\
-\left\langle\sigma_{-n}, u_{n}\right\rangle_{\Gamma_{11}}-\left\langle f-\alpha_{0} \theta f, u\right\rangle=\left\langle f_{1}, u\right\rangle, \quad \forall u \in L^{2}(0, T ; \mathbf{V}) \\
\left|\sigma_{\tau}\right| \leq g_{\tau}, \sigma_{\tau} \cdot v_{\tau}+g_{\tau}\left|v_{\tau}\right|=0 \quad \text { on } \Gamma_{8}, \\
\left|\sigma_{n}\right| \leq g_{n}, \sigma_{n} v_{n}+g_{n}\left|v_{n}\right|=0 \quad \text { on } \Gamma_{9}, \\
\sigma_{+n}+g_{+n} \geq 0,\left\langle\sigma_{+n}+g_{+n}, v_{n}\right\rangle_{\Gamma_{10}}=0 \quad \text { on } \Gamma_{10} \\
\sigma_{-n}-g_{-n} \leq 0,\left\langle\sigma_{-n}-g_{-n}, v_{n}\right\rangle_{\Gamma_{11}}=0 \quad \text { on } \Gamma_{11} .
\end{array}\right.
$$

Subtracting the first formula of (21) with $u=v$ from the first formula of (21), we get

For fixed $\theta$, let us consider the problem

$$
\begin{aligned}
& \left\langle\frac{\partial v}{\partial t}, u-v\right\rangle+a_{01}(v, u-v)+a_{11}(v, v, u-v)-\left(\sigma_{\tau}, u_{\tau}-v_{\tau}\right)_{\Gamma_{8}}-\left(\sigma_{n}, u_{n}-v_{n}\right)_{\Gamma_{9}}, \\
& \quad-\left\langle\sigma_{+n}, u_{n}-v_{n}\right\rangle_{\Gamma_{10}}-\left\langle\sigma_{-n}, u_{n}-v_{n}\right\rangle_{\Gamma_{11}}-\left\langle f-\alpha_{0} \theta f, u-v\right\rangle=\left\langle f_{1}, u-v\right\rangle, \quad \forall u \in \mathbf{V} .
\end{aligned}
$$

Define the functionals $\phi_{\tau}, \phi_{n}, \phi_{+}, \phi_{-}$, respectively, by

$$
\begin{aligned}
& \phi_{\tau}(\eta)=\int_{\Gamma_{8}} g_{\tau}|\eta| \mathrm{d} x, \quad \forall \eta \in \mathbf{L}_{\tau}^{2}\left(\Gamma_{8}\right), \\
& \phi_{n}(\eta)=\int_{\Gamma_{9}} g_{n}|\eta| \mathrm{d} x, \quad \forall \eta \in L^{2}\left(\Gamma_{9}\right), \\
& \phi_{+}(\eta)=\int_{\Gamma_{10}} g_{+n} \eta \mathrm{d} x, \quad \forall \eta \in L^{2}\left(\Gamma_{10}\right), \\
& \phi_{-}(\eta)=-\int_{\Gamma_{11}} g_{-n} \eta \mathrm{d} x, \quad \forall \eta \in L^{2}\left(\Gamma_{11}\right) .
\end{aligned}
$$

Since if $u \in K(\Omega)$, then $\left.u\right|_{\Gamma_{8}} \in \mathbf{L}_{\tau}^{2}\left(\Gamma_{8}\right),\left.u_{n}\right|_{\Gamma_{9}} \in L^{2}\left(\Gamma_{9}\right)$, $\left.u_{n}\right|_{\Gamma_{10}} \in L^{2}\left(\Gamma_{10}\right)$, and $\left.u_{n}\right|_{\Gamma_{11}} \in L^{2}\left(\Gamma_{11}\right)$, in what follows, for convenience, we use the notation

$$
\begin{aligned}
\phi_{\tau}(u)= & \phi_{\tau}\left(\left.u\right|_{\Gamma_{8}}\right), \\
\phi_{n}(u)= & \phi_{n}\left(\left.u_{n}\right|_{\Gamma_{9}}\right), \\
\phi_{+}(u)= & \phi_{+}\left(\left.u_{n}\right|_{\Gamma_{10}}\right), \\
\phi_{-}(u)= & \phi_{-}\left(\left.u_{n}\right|_{\Gamma_{11}}\right), \\
& \forall u \in K(\Omega) .
\end{aligned}
$$

Define a functional $\Phi: \mathbf{V} \longrightarrow \overline{\mathbb{R}} \equiv \mathbb{R} \cup\{+\infty\}$ by 


$$
\Phi(u)= \begin{cases}\phi_{\tau}(u)+\phi_{n}(u)+\phi_{+}(u)+\phi_{-}(u), & \forall u \in K(\Omega), \\ +\infty, & \forall u \notin K(\Omega) .\end{cases}
$$

Note that $\Phi(u) \geq 0$ since $\left.u_{n}\right|_{\Gamma_{10}} \geq 0,\left.u_{n}\right|_{\Gamma_{11}} \leq 0, \forall u \in K(\Omega)$. Then, the functional $\Phi \in(\mathbf{V} \longrightarrow \overline{\mathbb{R}}$ ) is proper (cf. Definition A.1 of [21]), convex, lower semicontinuous, and

$$
\Phi(u) \geq 0, \quad \forall u \in \mathbf{V}, \Phi\left(0_{V}\right)=0 .
$$

Define a functional $\Psi(u)$ by

$\Psi(u)= \begin{cases}\int_{0}^{T} \Phi(u(t)) \mathrm{d} t, & \text { if } \Phi(u(t)) \in L^{1}(0, T), \\ +\infty, & \text { otherwise. }\end{cases}$

In the same way as Problem I in [24], from (25), we get

$$
\begin{aligned}
& \left\langle\frac{\partial v}{\partial t}, u-v\right\rangle+a_{01}(v, u-v)+a_{11}(v, v, u-v)+\Phi(u)-\Phi(v) \\
& \quad \geq\left\langle\left(1-\alpha_{0} \theta\right) f, u-v\right\rangle+\left\langle f_{1}, u-v\right\rangle .
\end{aligned}
$$

Define operators $A_{1} \in\left(\mathbf{V} \longrightarrow \mathbf{V}^{*}\right), B_{1} \in(\mathbf{V} \times \mathbf{V} \longrightarrow$ $\left.\mathbf{V}^{*}\right)$, and $C_{1} \in\left(W_{\Gamma_{D}}^{1,2}(\Omega) \longrightarrow\left(W_{\Gamma_{D}}^{1,2}(\Omega)\right)^{*}\right)$, respectively, by

$$
\begin{aligned}
\left\langle A_{1} v, u\right\rangle & =a_{01}(v, u), \quad \forall v, u \in \mathbf{V}, \\
\left\langle B_{1}(v, u), w\right\rangle & =a_{11}(v, u, w), \quad \forall v, u, w \in \mathbf{V}, \\
\left\langle C_{1} \theta, \varphi\right\rangle & =b_{01}(\theta, \varphi), \quad \forall \theta, \varphi \in W_{\Gamma_{D}}^{1,2}(\Omega) .
\end{aligned}
$$

If $v$ is a solution to (24), then we can see that the solution satisfies (cf. (3 20) of [21])

$$
\int_{0}^{T}\left\langle v^{\prime}(t)+A_{1} v(t)+B_{1}(v(t), v(t))-\left(1-\alpha_{0}\right) \theta(t) f(t)-f_{1}(t), u(t)-v(t)\right\rangle \mathrm{d} t+\Psi(u)-\Psi(v) \geq 0, \quad \forall u \in L^{4}(0, T ; \mathbf{V})
$$

Therefore, we have the following variational formulation for problems (1), (4), and (5).

Problem (I-VI). Find $\quad(v, \theta) \in\left(L^{\infty}(0, T ; H) \cap\right.$ $\left.L^{2}(0, T ; \mathbf{V})\right) \times\left(L^{2}\left(0, T ; W_{\Gamma_{D}}^{1,2}\right) \cap L^{\infty}\left(0, T ; L^{2}\right)\right)$ such that

$$
\left\{\begin{array}{l}
\int_{0}^{T}\left\langle v^{\prime}+A_{1} v(t)+B_{1}(v(t), v(t))-\left(1-\alpha_{0} \theta\right) f-f_{1}, u(t)-v(t)\right\rangle \mathrm{d} t+\Psi(u)-\Psi(v) \geq 0, \quad \forall u \in L^{4}(0, T ; \mathbf{V}), \\
\int_{0}^{T}\left[\left\langle\frac{\partial \theta}{\partial t}, \varphi\right\rangle+\left\langle C_{1} \theta(t), \varphi\right\rangle-\langle\theta v, \nabla \varphi\rangle-\left\langle g_{1}, \varphi\right\rangle\right] \mathrm{d} t=0, \quad \forall \varphi \in L^{2}\left(0, T ; W_{\Gamma_{D}}^{1,2}\right), \\
v(0)=v_{0}, \\
\theta(0)=\theta_{0} .
\end{array}\right.
$$

Remark 4. If the solutions to Problem I-VI are smooth as much as $v \in L^{2}(0, T ; \mathbf{V})$ and $v^{\prime} \in L^{2}\left(0, T ; \mathbf{V}^{*}\right)$, then the first one of (34) is equivalent to

$$
\left\langle v^{\prime}(t)+A_{1} v(t)+B_{1}(v(t), v(t))-\left(1-\alpha_{0} \theta\right) f-f_{1}, u-v(t)\right\rangle+\Phi(t)-\Phi(v(t)) \geq 0, \quad \text { for a.e.t } \in[0, T], \forall u \in K(\Omega),
$$

(Remark, pp. 114 in [26]). In (31), putting $\left\langle F_{1}, u-v\right\rangle=$ $\left\langle-(\partial v / \partial t)-\left(1-\alpha_{0} \theta\right) f-f_{1}, u-v\right\rangle$, by Theorem 3.5 of [24], we can see existence of $\left(\sigma_{\tau}, \sigma_{n}, \sigma_{+n}, \sigma_{-n}\right) \in \mathbf{L}_{\tau}^{2}\left(\Gamma_{8}\right) \times L^{2}\left(\Gamma_{9}\right) \times$ $H^{-1 / 2}\left(\Gamma_{10}\right) \times H^{-1 / 2}\left(\Gamma_{11}\right)$ for a.e. $t \in(0, T)$ such that $\left(v, \theta, \sigma_{\tau}, \sigma_{n}, \sigma_{+n}, \sigma_{-n}\right)$ is a solution to Problem I-VE.
3.2. Variational Formulations: The Case of Total Pressure. Taking $(v \cdot \nabla) v=\operatorname{rot} v \times v+(1 / 2) \operatorname{grad}|v|^{2}$ into account, by (14)-(17) with $\mu, \kappa$ depending on $\theta$, we can see that smooth solutions $(v, p, \theta)$ of problems (1), (4), and (6) satisfy the following: 


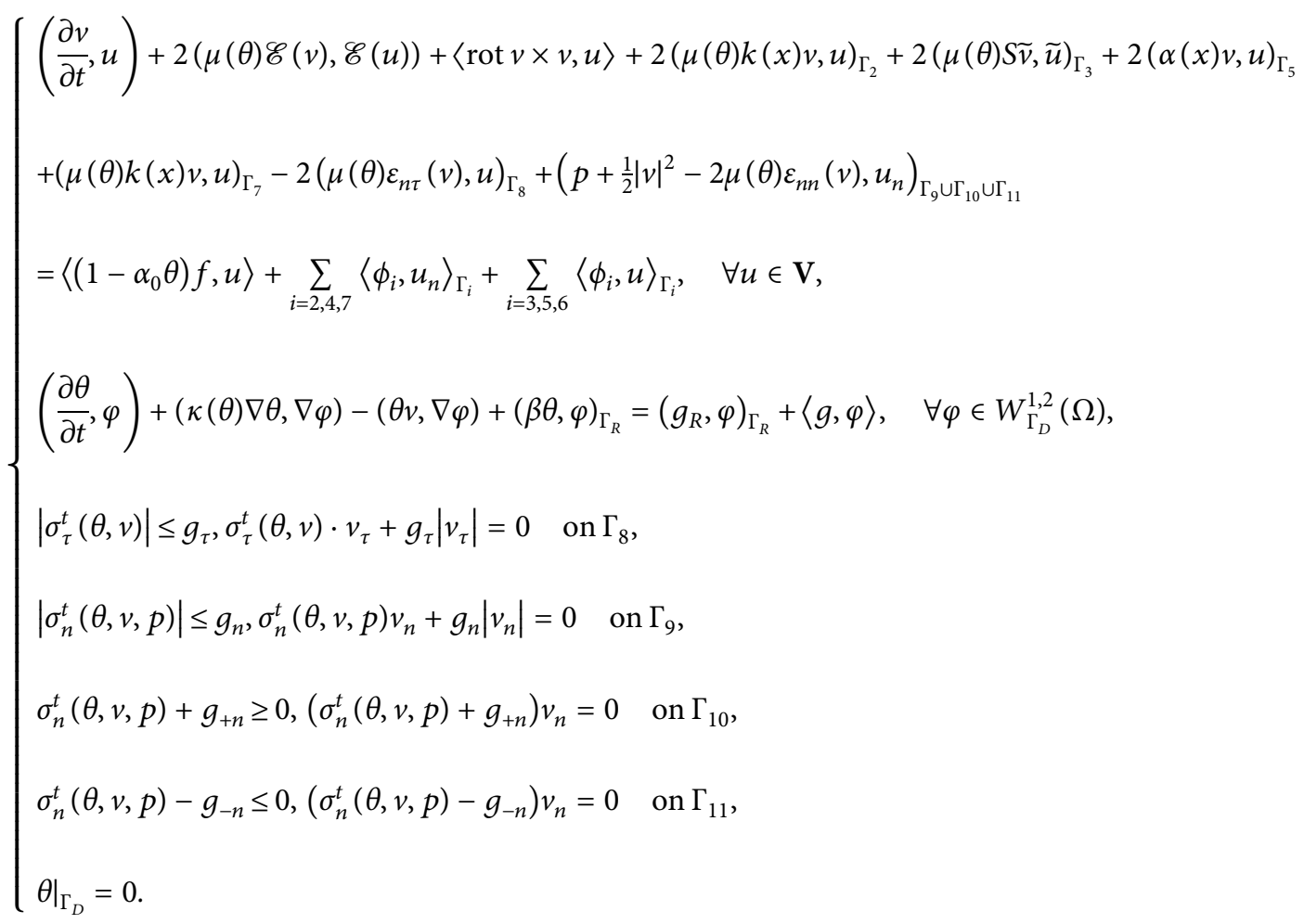

Define $a_{02}(\tilde{\theta} ; \cdot, \cdot), a_{12}(\cdot, \cdot, \cdot)$, and $f_{2} \in V^{*}$ by $a_{02}(\tilde{\theta} ; w, u)=2(\mu(\tilde{\theta}) \mathscr{E}(w), \mathscr{E}(u))+2(\mu(\tilde{\theta}) k(x) w, u)_{\Gamma_{2}}$

$$
\begin{aligned}
& +2(\mu(\tilde{\theta}) S \widetilde{w}, \widetilde{u})_{\Gamma_{3}}+2(\alpha(x) w, u)_{\Gamma_{5}} \\
& +(\mu(\tilde{\theta}) k(x) w, u)_{\Gamma_{7}},
\end{aligned}
$$$$
\forall w, u \in \mathbf{V}, \tilde{\theta} \in W_{\Gamma_{D}}^{1,2}(\Omega),
$$

$a_{12}(v, u, w)=\langle\operatorname{rot} v \times u, w\rangle, \quad \forall v, u, w \in \mathbf{V}$,

$$
\left\langle f_{2}, u\right\rangle=\sum_{i=2,4,7}\left\langle\phi_{i}, u_{n}\right\rangle_{\Gamma_{i}}+\sum_{i=3,5,6}\left\langle\phi_{i}, u\right\rangle_{\Gamma_{i}}, \quad \forall u \in \mathbf{V} .
$$

Define $b_{2}(\widetilde{\theta} ; \cdot, \cdot)$ and $g_{2} \in\left(W_{\Gamma_{D}}^{1,2}(\Omega)\right)^{*}$ by $b_{2}(\widetilde{\theta} ; \theta, \varphi)=(\kappa(\widetilde{\theta}) \nabla \theta, \nabla \varphi)+(\beta(x) \theta, \varphi)_{\Gamma_{R}}$,

$$
\forall \tilde{\theta}, \theta \in W_{\Gamma_{D}}^{1,2}(\Omega), \varphi \in W_{\Gamma_{D}}^{1,2}(\Omega),
$$

$$
\left\langle g_{2}, \varphi\right\rangle=\left(g_{R}, \varphi\right)_{\Gamma_{R}}+\langle g, \varphi\rangle, \quad \forall \varphi \in W_{\Gamma_{D}}^{1,2}(\Omega) .
$$

By (11) and (12),

$$
\begin{aligned}
& f_{2} \in L^{2}\left(0, T ; \mathbf{V}^{*}\right), \\
& g_{2} \in L^{2}\left(0, T ;\left(W_{\Gamma_{D}}^{1,2}\right)^{*}\right) .
\end{aligned}
$$

Then, taking into account

$$
\begin{aligned}
\sigma_{\tau}^{t}(\theta, v) & =2 \mu(\theta) \varepsilon_{n \tau}(v), \\
\sigma_{n}^{t}(\theta, v, p) & =-\left(p+\frac{1}{2}|v|^{2}\right)+2 \mu(\theta) \varepsilon_{n n}(v),
\end{aligned}
$$

and (36), we introduce the following variational formulation for problems (1), (4), and (6).

Problem (II-VE). Find $v \in K(Q), \quad \theta \in L^{\infty}\left(0, T ; L^{2}(\Omega)\right) \cap$ $L^{2}\left(0, T ; W_{\Gamma_{D}}^{1,2}(\Omega)\right), \quad$ and $\quad\left(\sigma_{\tau}^{t}, \sigma_{n}^{t}, \sigma_{+n}^{t}, \sigma_{-n}^{t}\right) \in \mathbf{L}_{\tau}^{2}\left(\Gamma_{8}\right) \times$ $L^{2}\left(\Gamma_{9}\right) \times H^{D}(1 / 2)\left(\Gamma_{10}\right) \times H^{-(1 / 2)}\left(\Gamma_{11}\right)$, in a.e., $t \in(0, T)$, such that $v(0)=v_{0}, \theta(0)=\theta_{0}$, and

$$
\left\{\begin{array}{l}
\left\langle\frac{\partial v}{\partial t}, u\right\rangle+a_{02}(\theta ; v, u)+a_{12}(v, v, u)-\left(\sigma_{\tau}^{t}, u_{\tau}\right)_{\Gamma_{8}}-\left(\sigma_{n}^{t}, u_{n}\right)_{\Gamma_{9}} \\
-\left\langle\sigma_{+n}^{t}, u_{n}\right\rangle_{\Gamma_{10}}-\left\langle\sigma_{-n}^{t}, u_{n}\right\rangle_{\Gamma_{11}}-\left\langle f-\alpha_{0} \theta f, u\right\rangle=\left\langle f_{2}, u\right\rangle, \quad \forall u \in \mathbf{V}, \\
\left\langle\frac{\partial \theta}{\partial t}, \varphi\right\rangle+b_{2}(\theta ; \theta, \varphi)-\langle\theta v, \nabla \varphi\rangle=\left\langle g_{2}, \varphi\right\rangle, \quad \forall \varphi \in W_{\Gamma_{D}}^{1,2}(\Omega), \\
\left|\sigma_{\tau}^{t}\right| \leq g_{\tau}, \sigma_{\tau}^{t} \cdot v_{\tau}+g_{\tau}\left|v_{\tau}\right|=0 \quad \text { on } \Gamma_{8}, \\
\left|\sigma_{n}^{t}\right| \leq g_{n}, \sigma_{n}^{t} v_{n}+g_{n}\left|v_{n}\right|=0 \quad \text { on } \Gamma_{9}, \\
\sigma_{+n}^{t}+g_{+n} \geq 0,\left\langle\sigma_{+n}^{t}+g_{+n}, v_{n}\right\rangle_{\Gamma_{10}}=0 \quad \text { on } \Gamma_{10}, \\
\sigma_{-n}^{t}-g_{-n} \leq 0,\left\langle\sigma_{-n}^{t}-g_{-n}, v_{n}\right\rangle_{\Gamma_{11}}=0 \quad \text { on } \Gamma_{11},
\end{array}\right.
$$

where $\mathbf{L}_{\tau}^{2}\left(\Gamma_{8}\right)$ is the subspace of $\mathbf{L}^{2}\left(\Gamma_{8}\right)$ consisting of functions such that $(u, n)_{\mathbf{L}^{2}\left(\Gamma_{8}\right)}=0$.

Define operators $A_{2}(\theta) \in\left(\mathbf{V} \longrightarrow \mathbf{V}^{*}\right)$ and $B_{2} \in\left(\mathbf{V} \times \mathbf{V} \longrightarrow \mathbf{V}^{*}\right)$, respectively, by 


$$
\begin{aligned}
\left\langle A_{2}(\tilde{\theta}) v, u\right\rangle & =a_{02}(\tilde{\theta} ; v, u), \quad \forall v, u \in \mathbf{V}, \tilde{\theta} \in W_{\Gamma_{D}}^{1,2}(\Omega), \\
\left\langle B_{2}(v, u), w\right\rangle & =a_{12}(v, u, v), \quad \forall v, u, w \in \mathbf{V} .
\end{aligned}
$$

Let functional $\Psi$ be defined by (25)-(28). Then, in the same way as Problem I-VI of [21], we find a variational inequality for velocity. Then, we get another variational formulation consisting of a variational inequality for velocity and a variational equation for temperature, which is equivalent to Problem II-VE if the solution is smooth enough.

Problem (II-VI). Find $(v, \theta) \in\left(L^{\infty}(0, T ; H) \cap L^{2}(0, T ; \mathbf{V})\right) \times$ $\left(L^{\infty}\left(0, T ; L^{2}(\Omega)\right) \cap L^{2}\left(0, T ; W_{\Gamma_{D}}^{1,2}(\Omega)\right)\right)$ such that

$$
\left\{\begin{array}{l}
\int_{0}^{T}\left\langle u^{\prime}+A_{2}(\theta) v(t)+B_{2}(v(t), v(t))-\left(1-\alpha_{0} \theta\right) f-f_{2}, u(t)-v(t)\right\rangle \mathrm{d} t+\Psi(u)-\Psi(v) \\
\geq-\frac{1}{2}\left\|v_{0}-u(0)\right\|^{2}, \quad \forall u \in L^{4}(0, T ; \mathbf{V}) \text { with } u^{\prime} \in L^{2}\left(0, T ; \mathbf{V}^{*}\right), \\
\int_{0}^{T}\left[\left\langle-\theta, \frac{\partial \varphi}{\partial t}\right\rangle+b_{2}(\theta ; \theta, \varphi)-\langle\theta v, \nabla \varphi\rangle-\left\langle g_{2}, \varphi\right\rangle\right] \mathrm{d} t \\
=\left\langle\theta_{0}(x), \varphi(x, 0)\right\rangle, \quad \forall \varphi \in C^{1}\left([0, T] ; W_{\Gamma_{D}}^{1,2}(\Omega)\right) \text { with } \varphi(\cdot, T)=0 .
\end{array}\right.
$$

3.3. Main Results. The main results of this paper are as follows.

Theorem 1 (the case of static pressure). Let Assumption 1 be satisfied. Suppose that

(1) The norms of $f, \phi_{i}, i=2-6, g, g_{R}$ in the spaces they belong to are small enough

(2) $v_{0} \in \mathbf{V}$ and $\Phi\left(v_{0}\right)=0$

(3) $\theta_{0} \in W_{\Gamma_{D}}^{1,2}(\Omega)$

(4) $\left(A_{1} v_{0}+B_{1}\left(v_{0}, v_{0}\right)-f_{1}(0)\right) \in H$ (compatibility condition at initial time for velocity)

(5) $\left\|v_{0}\right\|_{\mathrm{V}}$ and $\| A_{1} v_{0}+B_{1}\left(v_{0}, v_{0}\right)-\left(1-\alpha_{0} \theta_{0}\right) f(0)-$ $f_{1}(0) \|$ are small enough

(6) $\left(C_{1} \theta_{0}+v_{0} \cdot \nabla \theta_{0}-g_{1}(0)\right) \in L^{2}(\Omega) \quad$ (compatibility condition at initial time for temperature)

(7) $\left\|\theta_{0}\right\|_{W_{\Gamma}^{1,2}(\Omega)}$ and $\left\|C_{1} \theta_{0}+v_{0} \cdot \nabla \theta_{0}-g_{1}(0)\right\|_{L^{2}(\Omega)}$ are small enough

Then, there exists a solution $(v, \theta)$ to (34) such that

$$
\begin{aligned}
& v \in C([0, T] ; \mathbf{V}), \\
& v^{\prime} \in L^{2}(0, T ; \mathbf{V}) \cap L^{\infty}(0, T ; H), \\
& \theta \in C\left([0, T] ; W_{\Gamma_{D}}^{1,2}(\Omega)\right), \\
& \theta^{\prime} \in L^{2}\left(0, T ; W_{\Gamma_{D}}^{1,2}(\Omega)\right) \cap L^{\infty}\left(0, T ; L^{2}(\Omega)\right) .
\end{aligned}
$$

The solution satisfying $\|v\|_{\mathrm{V}} \leq c$ and $\|\theta\|_{W_{\Gamma_{D}}^{1,2}} \leq c$ for a constant $c>0$ small enough is unique.

Theorem 2 (the case of total pressure). Let Assumption 2 be satisfied; $v_{0} \in H_{K}$ and $\theta_{0} \in L^{2}(\Omega)$. Then, there exists a solution $\quad(v, \theta) \in\left(L^{\infty}(0, T ; H) \cap L^{2}(0, T ; \mathbf{V})\right) \times\left(L^{\infty}(0, T\right.$; $\left.\left.L^{2}(\Omega)\right) \cap L^{2}\left(0, T ; W_{\Gamma_{D}}^{1,2}(\Omega)\right)\right)$ to $(43)$.

\section{Proof of Theorem 1}

4.1. Existence and Estimation of Solutions to an Approximate Problem. We first consider a problem approximating (34). For every $0<\varepsilon<1$, define a functional $\Phi_{\varepsilon}$ by

$$
\Phi_{\varepsilon}(y)=\inf \left\{\frac{\|y-u\|_{\mathbf{V}}^{2}}{2 \varepsilon}+\Phi(u) ; u \in \mathbf{V}\right\}, \quad y \in \mathbf{V},
$$

which is called the Moreau regularization of $\Phi$. When $\partial \Phi: \mathbf{V} \longrightarrow 2^{\mathbf{V}}$ is the subdifferential of $\Phi$ in the Hilbert space $\mathbf{V}$, let $J_{\varepsilon}=(I+\varepsilon \partial \Phi)^{-1}$ and $(\partial \Phi)_{\varepsilon}:=\varepsilon^{-1}\left(I-J_{\varepsilon}\right)$ (the Yosida approximation of $\partial \phi$ ) for all $\varepsilon>0$. Then, the functional $\Phi_{\varepsilon}$ is convex, continuous, Fréchet differentiable, and $\nabla \Phi_{\varepsilon}=(\partial \Phi)_{\varepsilon} \equiv \varepsilon^{-1}\left(I-J_{\varepsilon}\right)$ for all $1>\varepsilon>0$. Moreover,

$$
\begin{gathered}
\Phi_{\varepsilon}(y)=\frac{\left\|y-J_{\varepsilon} y\right\|_{\mathbf{V}}^{2}}{2 \varepsilon}+\Phi\left(J_{\varepsilon} y\right), \quad \forall y \in \mathbf{V}, \\
\lim _{\varepsilon \longrightarrow 0} \Phi_{\varepsilon}(y)=\Phi(y), \quad \Phi\left(J_{\varepsilon} y\right) \leq \Phi_{\varepsilon}(y) \leq \Phi(y), \quad \forall y \in \mathbf{V},
\end{gathered}
$$

(cf. Theorem 2.9 in [27]). The operator $\nabla \Phi_{\varepsilon}$ is Lipschitz continuous with the constant $2 \varepsilon^{-1}$ (cf. Proposition 2.3 in [27]) and monotone (cf. Lemma 4.10 of ch. III in [25]).

By the fact that $\Gamma_{2 j}, \Gamma_{3 j}$, and $\Gamma_{7 j}$ are in $C^{2.1}\left(\Gamma_{i j}\right)$ and 4 of Assumption 1, there exists a constant $M$ such that

$$
\|S(x)\|_{\infty},\|k(x)\|_{\infty},\|\alpha\|_{L_{\infty}\left(\Gamma_{5}\right)} \leq M
$$

Thus, there exists $c_{*}$ such that 


$$
\begin{aligned}
& 2\left|\mu(k(x) z, z)_{\Gamma_{2}}+2 \mu(S \widetilde{z}, \widetilde{z})_{\Gamma_{3}}+(\alpha(x) z, z)_{\Gamma_{5}}+\mu(k(x) z, z)_{\Gamma_{7}}\right| \\
& \quad \leq \frac{\mu}{4}\|z\|_{\mathbf{V}}^{2}+c_{*}\|z\|^{2}, \quad \forall z \in \mathbf{V}
\end{aligned}
$$

(cf. .5.1.10 of [28]). Thus,

$$
\begin{gathered}
\left\langle A_{1} u, u\right\rangle \geq \frac{7 \mu}{4}\|u\|_{\mathbf{V}}^{2}-c_{*}\|u\|^{2}, \quad \forall u \in \mathbf{V}, \\
\left|\left\langle A_{1} u, v\right\rangle\right| \leq c_{1}\|u\|_{\mathbf{V}}\|v\|_{\mathrm{V}}, \quad \exists c_{1}>0, \forall u, v \in \mathbf{V}, \\
\left|\left\langle B_{1}(v, u), w\right\rangle\right| \leq c_{2}\|v\|_{\mathrm{V}}\|u\|_{\mathrm{V}}\|w\|_{\mathrm{V}}, \quad \forall u, v \in \mathbf{V},
\end{gathered}
$$

where the operators $A_{1}, B_{1}$ are the ones in (32).

Let $\left\{u_{j}, j=1,2, \ldots\right\}$ and $\left\{\varphi_{j}, j=1,2, \ldots\right\}$ be, respectively, bases of the space $\mathbf{V}$ and $W_{\Gamma_{D}}^{1,2}(\Omega)$. Without loss of generality, we assume that $u_{1}=v_{0}$ and $\varphi_{1}=\theta_{0}$ as in [26]. We find a solution $v_{m}=\sum_{j=1}^{m} g_{j m}(t) u_{j}$ and $\theta_{m}=\sum_{j=1}^{m} r_{j m}(t) \varphi_{j}$ to the problem

$$
\left\{\begin{array}{l}
\left\langle\frac{\partial v_{m}}{\partial t}, u_{j}\right\rangle+2\left\langle\mu \mathscr{E}\left(v_{m}\right), \mathscr{E}\left(u_{j}\right)\right\rangle+\left\langle\left(v_{m} \cdot \nabla\right) v_{m}, u_{j}\right\rangle+2\left(\mu k(x) v_{m}, u_{j}\right)_{\Gamma_{2}}+2\left(\mu S \widetilde{v}_{m}, \widetilde{u}_{j}\right)_{\Gamma_{3}} \\
+2\left(\alpha(x) v_{m}, u_{j}\right)_{\Gamma_{5}}+\left(\mu k(x) v_{m}, u_{j}\right)_{\Gamma_{7}}+\left\langle\nabla \Phi_{\varepsilon}\left(v_{m}(t)\right), u_{j}\right\rangle=\left\langle\left(1-\alpha_{0} \theta_{m}\right) f, u_{j}\right\rangle+\left\langle f_{1}, u_{j}\right\rangle \\
\left\langle\frac{\partial \theta_{m}}{\partial t}, \varphi_{j}\right\rangle+\left(\kappa \nabla \theta_{m}, \nabla \varphi_{j}\right)+\left(\beta(x) \theta_{m}, \varphi_{j}\right)_{\Gamma_{R}}-\left\langle v_{m} \theta_{m}, \nabla \varphi_{j}\right\rangle=\left\langle g_{1}, \varphi_{j}\right\rangle, \\
v_{m}(0)=v_{0} \\
\theta_{m}(0)=\theta_{0}
\end{array}\right.
$$

which gives us a system for $g_{j m}(t)$ and $r_{j m}(t), j=1-m$. The solutions to (52) depend on $\varepsilon$, but for convenience of notation, here and in what follows, we use subindex $m$ instead of subindex $m \varepsilon$. For $t_{m}$, there exist absolute continuous functions $g_{j m}(t)$ and $r_{j m}(t)$ on $\left[0, t_{m}\right)$. Since $f \in$ $W^{1, \infty}\left(0, T ; \mathbf{L}^{3}(\Omega)\right), f_{1} \in W^{1, \infty}\left(0, T ; \mathbf{V}^{*}\right), g_{1} \in W^{1,2}(0, T ;$ $\left.W_{\Gamma_{D}}^{1,2}(\Omega)^{*}\right)$, and $\nabla \Phi_{\varepsilon}$ is Lipschitz continuous, $g_{j m}^{\prime}(t)$ and $r_{j m}^{\prime}(t)$ are in fact absolute continuous. If $\left\|v_{m}(t)\right\|$ and $\left\|\theta_{m}(t)\right\|$ are bounded and $v_{m}(t), \theta_{m}(t)$ are integrable, then $g_{j m}(t)$ and $r_{j m}(t)$ are prolonged over $t_{m}$. Under smallness of the data of the problem and the compatibility condition of the data at the initial instant, we will find estimates for $\left\|v_{m}(t)\right\|$ and $\left\|\theta_{m}(t)\right\|$ in the following, by which we obtain (111) and see that $t_{m}=T$.

Multiplying the first and the second equation of (52), respectively, by $g_{j m}(t)$ and $\varphi_{j m}(t)$ and adding for $i=1, \ldots, m$, we get

$$
\left\{\begin{array}{l}
\left\langle\frac{\partial v_{m}}{\partial t}, v_{m}\right\rangle+2\left\langle\mu \mathscr{E}\left(v_{m}\right), \mathscr{E}\left(v_{m}\right)\right\rangle+\left\langle\left(v_{m} \cdot \nabla\right) v_{m}, v_{m}\right\rangle+2\left(\mu k(x) v_{m}, v_{m}\right)_{\Gamma_{2}}+2\left(\mu S \widetilde{v}_{m}, \widetilde{v}_{m}\right)_{\Gamma_{3}} \\
+2\left(\alpha(x) v_{m}, v_{m}\right)_{\Gamma_{5}}+\left(\mu k(x) v_{m}, v_{m}\right)_{\Gamma_{7}}+\left\langle\nabla \Phi_{\varepsilon}\left(v_{m}(t)\right), v_{m}\right\rangle=\left\langle\left(1-\alpha_{0} \theta_{m}\right) f, v_{m}\right\rangle+\left\langle f_{1}, v_{m}\right\rangle, \\
\left\langle\frac{\partial \theta_{m}}{\partial t}, \theta_{m}\right\rangle+\left(\kappa \nabla \theta_{m}, \nabla \theta_{m}\right)+\left(\beta(x) \theta_{m}, \theta_{m}\right)_{\Gamma_{R}}-\left\langle v_{m} \theta_{m}, \nabla \theta_{m}\right\rangle=\left\langle g_{1}, \theta_{m}\right\rangle, \\
v_{m}(0)=v_{0} \\
\theta_{m}(0)=\theta_{0} .
\end{array}\right.
$$


We will find a priori estimates for

$$
I(t):=\left\|v_{m}(t)\right\|^{2}+\left\|v_{m}^{\prime}(t)\right\|^{2}+\left\|\theta_{m}(t)\right\|^{2}+\left\|\theta_{m}^{\prime}(t)\right\|^{2} \text {. }
$$

Since $\Phi_{\varepsilon}$ is convex, continuous, and Fréchet differentiable, we have $\Phi_{\varepsilon}(u)-\Phi_{\varepsilon}\left(v_{m}(t)\right) \geq\left\langle\nabla \Phi_{\varepsilon}\left(v_{m}(t)\right), u-v_{m}(t)\right\rangle, \quad \forall u \in \mathbf{V}$,

and so by $\Phi_{\varepsilon}\left(0_{\mathbf{V}}\right)=0$,

$$
0 \leq \Phi_{\varepsilon}\left(v_{m}(t)\right) \leq\left\langle\nabla \Phi_{\varepsilon}\left(v_{m}(t)\right), v_{m}(t)\right\rangle
$$

Also,

$$
2\left|\left\langle-\alpha_{0} \theta_{m}(t) f, v_{m}(t)\right\rangle\right| \leq c\left|\alpha_{0}\right|\left\|\theta_{m}(t)\right\|_{W_{\Gamma_{D}}^{1,2}}^{2}\|f\|_{\mathbf{L}^{3}}^{2}+\frac{\mu}{4}\left\|v_{m}(t)\right\|_{\mathbf{V}}^{2}
$$

By virtue of (50), (51), (56), and (57), we have from the first equation of (53)

$$
\begin{aligned}
& \frac{\mathrm{d}}{\mathrm{d} t}\left\|v_{m}(t)\right\|^{2}+\frac{7 \mu}{2}\left\|v_{m}(t)\right\|_{\mathbf{V}}^{2}-2 c_{2}\left\|v_{m}(t)\right\|_{\mathbf{V}}^{3}+2 \Phi_{\varepsilon}\left(v_{m}(t)\right) \\
& \leq c\left|\alpha_{0}\right|\left\|\theta_{m}(t)\right\|_{W_{\Gamma_{D}}^{1,2}}^{2}\|f\|_{\mathbf{L}^{3}}^{2}+c\|f\|_{\mathbf{L}^{3}}^{2}+c\left\|f_{1}\right\|_{\mathbf{V}^{*}}^{2}+\frac{\mu}{2}\left\|v_{m}(t)\right\|_{\mathbf{V}}^{2}+2 c_{*}\left\|v_{m}(t)\right\|^{2},
\end{aligned}
$$

where $c_{*}$ and $c_{2}$ are, respectively, the ones in (49) and (51), and so

$$
\begin{aligned}
& \frac{\mathrm{d}}{\mathrm{d} t}\left\|v_{m}(t)\right\|^{2}+\left(3 \mu-2 c_{2}\left\|v_{m}(t)\right\|_{V}\right)\left\|v_{m}(t)\right\|_{V}^{2}+2 \Phi_{\varepsilon}\left(v_{m}(t)\right) \\
& \quad \leq c\left|\alpha_{0}\right|\left\|\theta_{m}(t)\right\|_{W_{\Gamma_{D}}^{1,2}}^{2}\|f\|_{L^{3}}^{2}+c\|f\|_{L^{3}}^{2}+c\left\|f_{1}\right\|_{V^{*}}^{2}+2 c_{*}\left\|v_{m}(t)\right\|^{2} .
\end{aligned}
$$

Here and in what follows are the constants independent of the data of problem which are denoted by $c$ with the exceptions of $c_{*}$ and $c_{2}$.

Setting $t=0$ in the first equation of (52) and multiplying the resulting equation by $g_{j m}^{\prime}(0)$ and adding for $j=1, \ldots, m$, we get

$$
\begin{aligned}
& \left\|v_{m}^{\prime}(0)\right\|^{2}+\left\langle A_{1} v_{m}(0), v_{m}^{\prime}(0)\right\rangle+\left\langle B_{1}\left(v_{m}(0), v_{m}(0)\right), v_{m}^{\prime}(0)\right\rangle \\
& \quad+\left\langle\nabla \Phi_{\varepsilon}\left(v_{0}\right), v_{m}^{\prime}(0)\right\rangle \\
& \quad=\left\langle\left(1-\alpha_{0} \theta_{0}\right) f(0), v_{m}^{\prime}(0)\right\rangle+\left\langle f_{1}(0), v_{m}^{\prime}(0)\right\rangle .
\end{aligned}
$$

By condition (2) of Theorem 1 , for any $u \in \mathbf{V}$, we have $\Phi(u) \geq \Phi\left(v_{0}\right)=0$, which by (47), it implies that $\Phi_{\varepsilon}\left(v_{0}\right)=0$ and $\nabla \Phi_{\varepsilon}\left(v_{0}\right)=0$. Then, from (60), we have

$$
\left\|v_{m}^{\prime}(0)\right\| \leq\left\|A_{1} v_{0}+B_{1}\left(v_{0}, v_{0}\right)-\left(1-\alpha_{0} \theta_{0}\right) f(0)-f_{1}(0)\right\|,
$$

which is valid by the compatibility condition at the initial time for velocity (condition (4)) and the conditions for $\theta_{0}, f$. On the contrary, taking into account (50), (51), and (56), we have from the first equation of (53)

$$
\begin{aligned}
\frac{7 \mu}{2}\left\|v_{m}(t)\right\|_{\mathrm{V}}^{2} \leq & 2 c_{2}\left\|v_{m}\right\|_{\mathrm{V}}^{3}+2\left\langle\left(1-\alpha_{0} \theta_{m}(t)\right) f(t), v_{m}(t)\right\rangle \\
& +2\left\langle f_{1}(t), v_{m}(t)\right\rangle+2 c_{*}\left\|v_{m}\right\|^{2}-2\left(v_{m}(t), v_{m}^{\prime}(t)\right),
\end{aligned}
$$

$$
\begin{aligned}
3 \mu\left\|v_{m}(t)\right\|_{\mathbf{V}} \leq & 2 c_{2}\left\|v_{m}(t)\right\|_{\mathbf{V}}^{2}+c\|f(t)\|_{\mathbf{L}^{3}}+c\left|\alpha_{0}\right|\left\|\theta_{m}(t)\right\|\|f(t)\|_{\mathbf{L}^{3}} \\
& +c\left\|f_{1}(t)\right\|_{\mathbf{V}^{*}}+\left(2 \delta\left\|v_{m}^{\prime}(t)\right\|+2 c_{*} \delta\left\|v_{m}(t)\right\|\right),
\end{aligned}
$$

where $\delta$ is such that $\|\cdot\| \leq \delta\|\cdot\|_{\mathbf{V}}$. Since $\left\langle v_{m} \theta_{m}, \nabla \theta_{m}\right\rangle=0$ by (17), we get from the second equation of (53)

$$
\frac{\mathrm{d}}{\mathrm{d} t}\left\|\theta_{m}(t)\right\|^{2}+2 \kappa\left\|\theta_{m}(t)\right\|_{W_{\Gamma_{D}}^{1,2}}^{2}+2\left(\beta(x) \theta_{m}, \theta_{m}\right)_{\Gamma_{R}}=2\left\langle g_{1}, \theta_{m}(t)\right\rangle \text {. }
$$

From (64), we have

$\left.\frac{\mathrm{d}}{\mathrm{d} t}\left\|\theta_{m}(t)\right\|^{2}+\kappa\left\|\theta_{m}(t)\right\|_{W_{\Gamma_{D}}^{1,2}}^{2}+\left(\beta(x) \theta_{m}, \theta_{m}\right)_{\Gamma_{R}} \leq \frac{1}{\kappa}\left\|g_{1}\right\|_{\left(W_{\Gamma_{D}}^{1,2}\right.}^{2}\right)^{*}$,

$$
\left\|\theta_{m}(t)\right\|^{2}+\int_{0}^{t} \int_{\Omega} \kappa\left|\nabla \theta_{m}(s)\right|^{2} \mathrm{~d} x \mathrm{~d} s \leq\left\|\theta_{0}\right\|^{2}+\frac{1}{\kappa} \int_{0}^{t}\left\|g_{1}(s)\right\|_{\left(W_{\Gamma_{D}}^{1,2}\right.}^{2} \mathrm{~d} s .
$$

Setting $t=0$ in the second equation of (52) and multiplying the resulting equation by $r_{j m}^{\prime}(0)$ and adding for $j=1, \ldots, m$, we get

$$
\left\|\theta_{m}^{\prime}(0)\right\|^{2}+b_{1}\left(\theta_{0}, \theta_{m}^{\prime}(0)\right)+\left(v_{0} \cdot \nabla \theta_{0}, \theta_{m}^{\prime}(0)\right)=\left\langle g(0), \theta_{m}^{\prime}(0)\right\rangle,
$$

where $-\left(v_{0} \theta_{0}, \nabla \theta_{m}^{\prime}(0)\right)=\left(v_{0} \cdot \nabla \theta_{0}, \theta_{m}^{\prime}(0)\right)$ was used. From (67), we have

$$
\left\|\theta_{m}^{\prime}(0)\right\| \leq\left\|C_{1} \theta_{0}+v_{0} \cdot \nabla \theta_{0}-g_{1}(0)\right\|,
$$

which is valid by the compatibility condition at the initial time for temperature (condition (6)).

On the contrary, taking into account $\left\langle v_{m} \theta_{m}, \nabla \theta_{m}\right\rangle=0$, from the second equation of (53), we have

$\left.\kappa\left\|\theta_{m}(t)\right\|_{W_{\Gamma_{D}}^{1,2}}^{2} \leq\left\|g_{1}\right\|_{\left(W_{\Gamma_{D}}^{1,2}\right.}\right)^{*}\left\|\theta_{m}(t)\right\|_{W_{\Gamma_{D}}^{1,2}}+\delta_{1}\left\|\theta_{m}(t)\right\|_{W_{\Gamma_{D}}^{1,2}}\left\|\theta_{m}^{\prime}(t)\right\|$,

and so 
where $\delta_{1}$ is such that $\|\cdot\| \leq \delta_{1}\|\cdot\|_{W_{\Gamma_{D}}^{1,2}}$, and so

$$
\left\|\theta_{m}(t)\right\|_{W_{\Gamma_{D}}^{1,2}} \leq \frac{1}{\mathcal{K}}\left(\left\|g_{1}\right\|_{\left(W_{\Gamma_{D}}^{1,2}\right)^{*}}+\delta_{1}\left\|\theta_{m}^{\prime}(t)\right\|\right) .
$$

Taking into account (66), we have from (63)

$$
\begin{aligned}
3 \mu\left\|v_{m}(t)\right\|_{\mathrm{V}} \leq & c_{2}\left\|v_{m}(t)\right\|_{\mathrm{V}}^{2}+c\|f\|_{L^{\infty}\left(0, T ; \mathbf{L}^{3}\right)} \\
& +c\left|\alpha_{0}\right|\left(\left\|\theta_{0}\right\|^{2}+\frac{1}{\mathcal{K}} \int_{0}^{T}\left\|g_{1}\right\|_{\left.\left(W_{\mathrm{I}_{D}}^{1,2}\right)^{*} \mathrm{~d} s\right)^{1 / 2}\|f\|_{L^{\infty}\left(0, T ; \mathbf{L}^{3}\right)}}\right. \\
& +\left\|f_{1}\right\|_{L^{\infty}\left(0, T ; \mathbf{V}^{*}\right)}+\max \left\{2 \delta, 2 c_{*} \delta\right\}\left(\left\|v_{m}^{\prime}(t)\right\|+\left\|v_{m}(t)\right\|\right) .
\end{aligned}
$$

Differentiating the first equality of (52) with respect to $t$, we have that

$$
\begin{aligned}
\left\langle v_{m}^{\prime \prime}\right. & \left.(t), v_{j}\right\rangle+\left\langle A_{1} v_{m}^{\prime}(t), v_{j}\right\rangle+\left\langle\left(B_{1}\left(v_{m}(t), v_{m}(t)\right)^{\prime}, v_{j}\right\rangle\right. \\
& +\left\langle\left(\nabla \Phi_{\varepsilon}\left(v_{m}\right)^{\prime}\right), v_{j}\right\rangle \\
& =\left\langle-\alpha_{0} \theta_{m}^{\prime}(t) f, v_{j}\right\rangle-\left\langle\left(1-\alpha_{0} \theta_{m}(t)\right) f^{\prime}, v_{j}\right\rangle+\left\langle f_{1}^{\prime}, v_{j}\right\rangle .
\end{aligned}
$$

Multiplying (72) by $g_{j m}^{\prime}(t)$ and adding for $j$, we have

$$
\begin{aligned}
& \left\langle v_{m}^{\prime \prime}(t), v_{m}^{\prime}(t)\right\rangle+\left\langle A_{1} v_{m}^{\prime}(t), v_{m}^{\prime}(t)\right\rangle+\left\langle\left(B_{1} v_{m}(t), v_{m}(t)\right)^{\prime}, v_{m}^{\prime}(t)\right\rangle+\left\langle\left(\nabla \Phi_{\varepsilon}\left(v_{m}\right)\right)^{\prime}, v_{m}^{\prime}(t)\right\rangle \\
& \quad=\left\langle-\alpha_{0} \theta_{m}^{\prime}(t) f, v_{m}^{\prime}(t)\right\rangle-\left\langle\left(1-\alpha_{0} \theta_{m}(t)\right) f \prime, v_{m}^{\prime}(t)\right\rangle+\left\langle f_{1}^{\prime}, v_{m}^{\prime}(t)\right\rangle .
\end{aligned}
$$

Calculating $\left(B\left(v_{m}(t), v_{m}(t)\right)\right)^{\prime}$, we have $\left|\left\langle\left(B_{1} v_{m}(t), v_{m}(t)\right)^{\prime}, v_{m}^{\prime}(t)\right\rangle\right|=\mid\left\langle B_{1}\left(v_{m}^{\prime}, v_{m}\right), v_{m}^{\prime}\right\rangle$

$$
+\left\langle B_{1}\left(v_{m}, v_{m}^{\prime}\right), v_{m}^{\prime}\right\rangle \mid \leq 2 c_{2}\left\|v_{m}\right\|_{\mathrm{V}}\left\|v_{m}^{\prime}\right\|_{\mathrm{V}}^{2},
$$

where $c_{2}$ is the one in (51). Also, by the Hölder inequality and the Young inequality, we have

$$
\begin{aligned}
2\left|\left\langle\alpha_{0} \theta_{m}^{\prime}(t) f, v_{m}^{\prime}(t)\right\rangle\right| & \leq c \mid \alpha_{0}\left\|\theta_{m}^{\prime}(t)\right\|_{W_{\mathrm{I}_{D}}^{1,2}}^{2}\|f\|_{\mathrm{L}^{3}}^{2}+\frac{\mu}{8}\left\|v_{m}^{\prime}(t)\right\|_{V}^{2}, \\
2\left|\left\langle\alpha_{0} \theta_{m}(t) f^{\prime}, v_{m}^{\prime}(t)\right\rangle\right| & \leq c\left|\alpha_{0}\right|\left\|\theta_{m}(t)\right\|_{W_{\mathrm{I}_{D}}^{1,2}}^{2}\left\|f^{\prime}\right\|_{\mathrm{L}^{3}}^{2}+\frac{\mu}{8}\left\|v_{m}^{\prime}(t)\right\|_{V^{2}}^{2}, \\
2\left|\left\langle f^{\prime}(t), v_{m}^{\prime}(t)\right\rangle\right| & \leq c\left\|f^{\prime}(t)\right\|_{\mathrm{L}^{3}}^{2}+\frac{\mu}{8}\left\|v_{m}^{\prime}(t)\right\|_{\mathrm{V}^{\prime}}^{2}, \\
2\left|\left\langle f_{1}^{\prime}(t), v_{m}^{\prime}(t)\right\rangle\right| & \leq c\left\|f_{1}^{\prime}(t)\right\|_{\mathbf{V}^{*}}^{2}+\frac{\mu}{8}\left\|v_{m}^{\prime}(t)\right\|_{\mathbf{V}^{\prime}}^{2}
\end{aligned}
$$

Taking into account (50), (74), and (75) and the fact that $\left\langle\left(\nabla \Phi_{\varepsilon}\left(v_{m}\right)\right)^{\prime}, v_{m}^{\prime}\right\rangle \geq 0$, which is owing to monotonicity of $r$ from $\nabla \Phi_{\varepsilon}$ (cf. [26], pp. 116), from (73), we have

$$
\begin{aligned}
& \frac{\mathrm{d}}{\mathrm{d} t}\left\|v_{m}^{\prime}(t)\right\|^{2}+\left(3 \mu-4 c_{2}\left\|v_{m}(t)\right\|_{\mathbf{V}}\right)\left\|v_{m}^{\prime}(t)\right\|_{\mathbf{V}}^{2}+\frac{\mu}{2}\left\|v_{m}^{\prime}(t)\right\|_{\mathbf{V}}^{2} \\
& \quad \leq c \alpha_{0}\left\|\theta_{m}^{\prime}(t)\right\|_{W_{\Gamma_{D}}^{1,2}}^{2}\|f\|_{\mathbf{L}^{3}}^{2}+c \mid \alpha_{0}\left\|\theta_{m}(t)\right\|_{W_{\Gamma_{D}}^{1,2}}^{2}\left\|f^{\prime}\right\|_{\mathbf{L}^{3}}^{2}+c\left\|f^{\prime}\right\|_{\mathbf{L}^{3}}^{2}+c\left\|f_{1}^{\prime}\right\|_{\mathbf{V}^{*}}^{2}+2 c_{*}\left\|v_{m}^{\prime}(t)\right\|^{2}+\frac{\mu}{2}\left\|v_{m}^{\prime}(t)\right\|_{\mathbf{V}^{\prime}}^{2}
\end{aligned}
$$

that is, 


$$
\begin{aligned}
& \frac{\mathrm{d}}{\mathrm{d} t}\left\|v_{m}^{\prime}(t)\right\|^{2}+\left(3 \mu-4 c_{2}\left\|v_{m}(t)\right\|_{\mathrm{V}}\right)\left\|v_{m}^{\prime}(t)\right\|_{\mathrm{V}}^{2} \\
& \quad \leq c\left|\alpha_{0}\left\|\theta_{m}^{\prime}(t)\right\|_{W_{\Gamma_{D}}^{1,2}}^{2}\|f\|_{\mathrm{L}^{3}}^{2}+c\right| \alpha_{0}\left\|\theta_{m}(t)\right\|_{W_{\Gamma_{D}}^{1,2}}^{2}\left\|f^{\prime}\right\|_{\mathbf{L}^{3}}^{2}+c\left\|f^{\prime}\right\|_{\mathrm{L}^{3}}^{2}+c\left\|f_{1}^{\prime}\right\|_{\mathbf{V}^{*}}^{2}+2 c_{*}\left\|v_{m}^{\prime}(t)\right\|^{2} .
\end{aligned}
$$

Differentiating the second equality of (52) with respect to $t$, we have that

$$
\begin{aligned}
& \left\langle\theta_{m}^{\prime \prime}(t), \varphi_{j}\right\rangle+\left\langle C_{1} \theta_{m}^{\prime}(t), \varphi_{j}\right\rangle-\left\langle v_{m}^{\prime} \theta, \nabla \varphi_{j}\right\rangle-\left\langle v_{m} \theta \prime, \nabla \varphi_{j}\right\rangle \\
& \quad=\left\langle g_{1}^{\prime}, \varphi_{j}\right\rangle .
\end{aligned}
$$

Multiplying (78) by $r_{j m}^{\prime}(t)$ and adding for $j$, we have that

$$
\begin{aligned}
& \left\langle\theta_{m}^{\prime \prime}(t), \theta_{m}^{\prime}(t)\right\rangle+\left\langle C_{1} \theta_{m}^{\prime}(t), \theta_{m}^{\prime}(t)\right\rangle-\left\langle v_{m}^{\prime} \theta_{m}, \nabla \theta_{m}^{\prime}(t)\right\rangle \\
& \quad-\left\langle v_{m} \theta I, \nabla \theta_{m}^{\prime}(t)\right\rangle=\left\langle g_{1}^{\prime}, \theta_{m}^{\prime}(t)\right\rangle .
\end{aligned}
$$

On the contrary, we get

$$
2\left|\left\langle v_{m}^{\prime} \theta, \nabla \theta_{m}^{\prime}(t)\right\rangle\right| \leq \frac{c}{\kappa}\left\|v_{m}^{\prime}\right\|_{V_{\mathrm{V}}}^{2}\|\theta\|_{W_{\mathrm{\Gamma}_{D}}^{1,2}}^{2}+\kappa\left\|\theta_{m}^{\prime}(t)\right\|_{W_{\mathrm{I}_{D}}^{1,2}}^{2} .
$$
Taking into account $\left\langle v_{m} \theta \prime, \nabla \theta_{m}^{\prime}(t)\right\rangle=0$ (see (17) and
$(80)$ ), we have from (79)

$$
\begin{aligned}
\frac{\mathrm{d}}{\mathrm{d} t}\left\|\theta_{m}^{\prime}(t)\right\|^{2}+2 \kappa\left\|\theta_{m}^{\prime}(t)\right\|_{W_{\Gamma_{D}}^{1,2}}^{2} & \frac{c}{\kappa}\left\|v_{m}^{\prime}\right\|_{V}^{2}\|\theta\|_{W_{\Gamma_{D}}^{1,2}}^{2}+\kappa\left\|\theta_{m}^{\prime}(t)\right\|_{W_{\Gamma_{D}}^{1,2}}^{2} \\
& \left.+c\left\|g_{1}^{\prime}\right\|_{\left(W_{\Gamma_{D}}^{1,2}\right.}^{2}\right)^{*}+\frac{\kappa}{4}\left\|\theta_{m}^{\prime}(t)\right\|_{W_{\Gamma_{D}}^{1,2}}^{2} .
\end{aligned}
$$

We have from (81)

$$
\frac{\mathrm{d}}{\mathrm{d} t}\left\|\theta_{m}^{\prime}(t)\right\|^{2}+\frac{3 \kappa}{4}\left\|\theta_{m}^{\prime}(t)\right\|_{W_{\Gamma_{D}}^{1,2}}^{2} \leq \frac{c}{\kappa}\left\|v_{m}^{\prime}\right\|_{\mathrm{V}^{\prime}}^{2}\|\theta\|_{W_{\Gamma_{D}}^{1,2}}^{2}+c\left\|g_{1}^{\prime}\right\|_{\left(W_{\Gamma_{D}}^{1,2}\right.}^{2}{ }^{*} .
$$

Adding (59), (77), (65), and (82), we have

$$
\begin{aligned}
& \frac{\mathrm{d}}{\mathrm{d} t} I(t)+\left(2 \mu-4 c_{2}\left\|v_{m}(t)\right\|_{\mathrm{V}}\right)\left(\left\|v_{m}(t)\right\|_{\mathrm{V}}^{2}+\left\|v_{m}^{\prime}(t)\right\|_{\mathbf{V}}^{2}\right)+\left(\mu-\frac{c}{\kappa}\left\|\theta_{m}\right\|_{W_{\Gamma_{D}}^{1,2}}^{2}\right)\left\|v_{m}^{\prime}(t)\right\|_{\mathbf{V}}^{2} \\
& \quad+\left(\kappa-c\left|\alpha_{0}\right|\|f\|_{\mathbf{L}^{3}}^{2}-c \mid \alpha_{0}\|f\|_{\mathbf{L}^{3}}^{2}\right)\left\|\theta_{m}\right\|_{W_{\Gamma_{D}}^{1,2}}^{2}+\left(\frac{3 \kappa}{4}-c\left|\alpha_{0}\right|\|f\|_{\mathbf{L}^{3}}^{2}\right)\left\|\theta_{m}^{\prime}\right\|_{W_{\Gamma_{D}}^{1,2}}^{2} \\
& \left.\quad \leq c\left(\|f\|_{\mathrm{L}^{3}}^{2}+\|f\|_{\mathrm{L}^{3}}^{2}\right)+c\left(\left\|f_{1}\right\|_{\mathbf{V}^{*}}^{2}+\left\|f_{1^{\prime}}^{\prime}\right\|_{\mathrm{V}^{*}}^{2}\right)+c\left(\left\|g_{1}\right\|_{\left(W_{\Gamma_{D}}^{1,2}\right)^{*}}^{2}+\left\|g_{1}^{\prime}\right\|_{\left(W_{\Gamma_{D}}^{1,2}\right.}^{2}\right)^{*}\right) \\
& \quad+2 c_{*}\left(\left\|v_{m}(t)\right\|^{2}+\left\|v_{m}^{\prime}(t)\right\|^{2}\right) .
\end{aligned}
$$

Integrating (83), we have

$$
\begin{aligned}
& I(t)+\int_{0}^{t}\left[\left(2 \mu-4 c_{2}\left\|v_{m}(s)\right\|_{\mathrm{V}}\right)\left(\left\|v_{m}(t)\right\|_{\mathrm{V}}^{2}+\left\|v_{m}^{\prime}(s)\right\|_{\mathrm{V}}^{2}\right)+\left(\mu-\frac{c}{\kappa}\left\|\theta_{m}\right\|_{W_{\Gamma_{D}}^{1,2}}^{2}\right)\left\|v_{m}^{\prime}(s)\right\|_{\mathrm{V}}^{2}+\left(\kappa-c\left|\alpha_{0}\right|\|f\|_{\mathbf{L}^{3}}^{2}-c\left|\alpha_{0}\right|\left\|f^{\prime}\right\|_{\mathbf{L}^{3}}^{2}\right)\left\|\theta_{m}\right\|_{W_{\mathrm{I}_{D}}^{1,2}}^{2}\right. \\
& \left.\quad+\left(\frac{3 \kappa}{4}-c\left|\alpha_{0}\right|\|f\|_{\mathbf{L}^{3}}^{2}\right)\left\|\theta_{m}^{\prime}\right\|_{W_{\mathrm{I}_{D}}^{1,2} \mathrm{~d} s}^{2}\right] \\
& \quad \leq I(0)+F(t)+2 c_{*} \int_{0}^{t}\left(\left\|v_{m}(s)\right\|^{2}+\left\|v_{m}^{\prime}(s)\right\|^{2}\right) \mathrm{d} s
\end{aligned}
$$

where

$$
\begin{aligned}
F(t):= & c t\left(\|f\|_{W^{1, \infty}\left(0, T ; \mathbf{L}^{3}\right)}^{2}+\left\|f_{1}\right\|_{W^{1, \infty}\left(0, T ; \mathbf{V}^{*}\right)}^{2}\right) \\
& +c\left\|g_{1}\right\|_{W^{1,2}\left(0, t ;\left(W_{\Gamma_{D}}^{1,2}\right)^{*}\right)}^{2} .
\end{aligned}
$$

By (61) and (68), we have

$$
\begin{aligned}
I(0) \leq & \left\|v_{0}\right\|^{2}+\left\|A_{1} v_{0}+B_{1}\left(v_{0}, v_{0}\right)-\left(1-\alpha_{0} \theta_{0}\right) f(0)-f_{1}(0)\right\|^{2} \\
& +\left\|\theta_{0}\right\|^{2}+\left\|C_{1} \theta_{0}+v_{0} \cdot \nabla \theta_{0}-g_{1}(0)\right\|^{2} .
\end{aligned}
$$


By the condition of theorem, let $\|f\|_{W^{1, \infty}\left(0, T ; \mathbf{L}^{3}\right)}$ be so small that

$$
\begin{array}{r}
\kappa-c\left|\alpha_{0}\right|\|f(t)\|_{\mathbf{L}^{3}}^{2}-c\left|\alpha_{0}\right|\left\|f^{\prime}(t)\right\|_{\mathbf{L}^{3}}^{2} \geq 0, \quad \text { at a.e. } t \in[0, T], \\
\frac{3 \kappa}{4}-c\left|\alpha_{0}\right|\|f\|_{\mathbf{L}^{3}}^{2} \geq 0, \quad \text { at a.e. } t \in[0, T] .
\end{array}
$$

If

$$
\begin{gathered}
\left\|v_{m}(0)\right\|_{\mathrm{V}}=\left\|v_{0}\right\|_{\mathrm{V}}<\frac{\mu}{2 c_{2}}, \\
\left\|\theta_{m}(0)\right\|_{{W_{\Gamma_{D}}^{1,2}}^{1,2}}=\left\|\theta_{0}\right\|_{W_{\Gamma_{D}}^{1,2}<\frac{\mu \kappa}{c},}
\end{gathered}
$$

are valid, then there exists $t_{m}$ such that

$$
\begin{aligned}
& 2 \mu-4 c_{2}\left\|v_{m}(t)\right\|_{\mathbf{V}} \geq 0, \\
& \mu-\frac{c}{\mathcal{K}}\left\|\theta_{m}(t)\right\|_{W_{\mathrm{I}_{D}}^{1,2}} \geq 0,
\end{aligned}
$$

on $\left[0, t_{m}\right]$. Therefore, taking into account (86), by the Gronwall inequality, we have

$$
\begin{aligned}
I(t) \leq & \left(\left\|v_{0}\right\|^{2}+\left\|A_{1} v_{0}+B_{1}\left(v_{0}, v_{0}\right)-\left(1-\alpha_{0} \theta_{0}\right) f(0)-f_{1}(0)\right\|^{2}\right. \\
& \left.+\left\|\theta_{0}\right\|^{2}+\left\|C_{1} \theta_{0}+v_{0} \cdot \nabla \theta_{0}-g_{1}(0)\right\|^{2}+F(T)\right) e^{2 c_{*} t},
\end{aligned}
$$

on all intervals of $t$ satisfying (90).

Using the estimate, we will obtain a quadratic inequality satisfied by $\left\|v_{m}(t)\right\|_{\mathrm{V}}$.

Put

$$
\begin{aligned}
\beta:= & \left(\left\|v_{0}\right\|^{2}+\left\|A_{1} v_{0}+B_{1}\left(v_{0}, v_{0}\right)-\left(1-\alpha_{0} \theta_{0}\right) f(0)-f_{1}(0)\right\|^{2}\right. \\
& \left.+\left\|\theta_{0}\right\|^{2}+\left\|C_{1} \theta_{0}+v_{0} \cdot \nabla \theta_{0}-g_{1}(0)\right\|^{2}+F(T)\right) e^{2 c_{*} T}
\end{aligned}
$$

Note that $\beta$ depends only on the data of the problem. Then, when $f$ satisfies (87), we can see from (91) that

$$
\begin{aligned}
\left(\left\|v_{m}(t)\right\|+\left\|v_{m}^{\prime}(t)\right\|\right) & \leq \sqrt{2}\left(\left\|v_{m}(t)\right\|^{2}+\left\|v_{m}^{\prime}(t)\right\|^{2}\right)^{1 / 2} \leq \sqrt{2 \beta}, \\
\left\|\theta_{m}^{\prime}(t)\right\| & \leq \sqrt{\beta},
\end{aligned}
$$

on $\left[0, t_{m}\right]$, where $(90)$ holds. Let the data of the problem be so small that

$$
\frac{c}{\kappa^{2}}\left(\left\|g_{1}\right\|_{W^{1,2}\left(0, T ;\left(W_{\Gamma_{D}}^{1,2}\right)^{*}\right)}+\delta_{1} \sqrt{\beta}\right) \leq \frac{\mu}{2}
$$
have

By (70) and (93), for the small data of the problem, we

$$
\frac{c}{\kappa}\left\|\theta_{m}(t)\right\|_{W_{\Gamma_{D}}^{1,2}} \leq \frac{c}{\mathcal{K}^{2}}\left(\left\|g_{1}\right\|_{W^{1,2}\left(0, T ;\left(W_{\Gamma_{D}}^{1,2}\right)\right)^{*}}+\delta_{1} \sqrt{\beta}\right) \leq \frac{\mu}{2},
$$

on $\left[0, t_{m}\right]$, which implies

$$
\mu-\frac{c}{\kappa}\left\|\theta_{m}(t)\right\|_{W_{\Gamma_{D}}^{1,2}} \geq \frac{\mu}{2}, \quad \forall t \in\left[0, t_{m}\right] .
$$

Therefore, for such small data of the problem that (94) is valid, if

$$
2 \mu-4 c_{2}\left\|v_{m}(t)\right\|_{\mathrm{V}} \geq 0, \quad \forall t \in\left[0, t_{m}+\gamma\right], \gamma>0, t_{m}+\gamma \leq T,
$$

then owing to (96), step by step, we have

$$
\mu-\frac{c}{\kappa}\left\|\theta_{m}(t)\right\|_{W_{\Gamma_{D}}^{1,2}} \geq \frac{\mu}{2}, \quad \forall t \in\left[0, t_{m}+\gamma\right] .
$$

From the above, we see that, for the small data of the problem satisfying (87)-(89) and (94),

$$
\mu-\frac{c}{\kappa}\left\|\theta_{m}(t)\right\|_{W_{\Gamma_{D}}^{1,2}} \geq \frac{\mu}{2}
$$

is valid on the interval where the first inequality of (90) is valid.

Put

$$
\begin{aligned}
\gamma:= & \|f(t)\|_{L^{\infty}\left(0, T ; \mathbf{L}^{3}\right)} \\
& +c\left|\alpha_{0}\right|\left(\left\|\theta_{0}\right\|^{2}+\frac{1}{\kappa} \int_{0}^{T}\left\|g_{1}\right\|_{\left(W_{\Gamma_{D}}^{1,2}\right)}^{2} \mathrm{~d} s\right)^{1 / 2}\|f(t)\|_{L^{\infty}\left(0, T ; \mathbf{L}^{3}\right)} \\
& +\left\|f_{1}(t)\right\|_{L^{\infty}\left(0, T ; V^{*}\right)}+\max \left\{2 \delta, 2 c_{*} \delta\right\} \sqrt{2 \beta} .
\end{aligned}
$$

By (66), (93), and (63), for the small data satisfying (87)-(89) and (94), we have a quadratic inequality for $\left\|v_{m}(t)\right\|_{\mathrm{V}}$, which is the one we want,

$$
0 \leq \gamma-3 \mu\left\|v_{m}(t)\right\|_{\mathrm{V}}+2 c_{2}\left\|v_{m}(t)\right\|_{\mathrm{V}}^{2}
$$

on the intervals where the first inequality of (90) is satisfied.

By the conditions of the theorem, we can assume that the data of the problem are so small that (87)-(89) and (94) are valid, and $\gamma$ satisfies the following inequality:

$$
9 \mu^{2}-8 c_{2} \gamma>4 \mu^{2} \text {. }
$$

Now, let us prove that if

$$
\left\|v_{0}\right\|_{\mathrm{V}} \leq \frac{3 \mu-\sqrt{9 \mu^{2}-8 c_{2} \gamma}}{4 c_{2}}\left(<\frac{\mu}{4 c_{2}}\right),
$$

then for any $m$,

$$
2 \mu-4 c_{2}\left\|v_{m}(t)\right\|_{\mathrm{V}} \geq \mu, \quad \forall t \in[0, T] .
$$

Since $2 \mu-4 c_{2}\left\|v_{0}\right\|_{\mathbf{V}}>\mu$, on an interval $\left[0, t_{m}\right]$,

$$
2 \mu-4 c_{2}\left\|v_{m}(t)\right\|_{\mathrm{V}} \geq \mu \text {. }
$$

Let us first prove that if the first inequality of (90) is valid on an interval $\left[0, \bar{t}_{m}\right]$, then more stronger 


$$
2 \mu-4 c_{2}\left\|v_{m}(t)\right\|_{\mathbf{V}} \geq \mu, \quad \forall t \in\left[0, \bar{t}_{m}\right],
$$

is valid. Putting $y=\left\|v_{m}(t)\right\|_{\mathbf{V}}$ in (101) (which is valid on the interval where the first inequality of (90) holds when (87)-(89) and (94) are valid), we get

$$
0 \leq \gamma-3 \mu y+2 c_{2} y^{2} \quad \text { on }\left[0, \bar{t}_{m}\right]
$$

By virtue of (102), there exist two real roots of $z=\gamma-3 \mu y+c_{2} y^{2}$ :

$$
\begin{aligned}
& y_{1}=\frac{3 \mu-\sqrt{9 \mu^{2}-8 c_{2} \gamma}}{4 c_{2}}, \\
& y_{2}=\frac{3 \mu+\sqrt{9 \mu^{2}-8 c_{2} \gamma}}{4 c_{2}},
\end{aligned}
$$

and on the intervals $\left[0, y_{1}\right]$ and $\left[y_{2},+\infty\right)$, (107) holds. Thus, by continuity of $\left\|v_{m}(t)\right\|_{\mathbf{v}}$ with respect to $t$ from $\|v(0)\|_{\mathbf{V}} \in\left[0, y_{1}\right]$, we have that $\left\|v_{m}(t)\right\|_{\mathbf{V}} \in\left[0, y_{1}\right] \forall t \in$ $\left[0, \bar{t}_{m}\right]$, that is,

$$
\left\|v_{m}(t)\right\|_{\mathbf{V}} \leq \frac{3 \mu-\sqrt{9 \mu^{2}-8 c_{2} \gamma}}{4 c_{2}}<\frac{\mu}{4 c_{2}}, \quad \forall t \in\left[0, \bar{t}_{m}\right] .
$$

Thus,

$$
2 \mu-4 c_{2}\left\|v_{m}(t)\right\|_{\mathbf{V}}>\mu, \quad \forall t \in\left[0, \bar{t}_{m}\right],
$$

which shows (106). Thus, by step by step, we see that the fist inequality of (90) is valid on $[0, T]$, and (104) is valid.

If (103) is valid, then so is (88). Therefore, for the small data satisfying (87), (89), (94), (102), and (103), we also have (99) on $[0, T]$. By (104) and (99), we have

$$
\begin{aligned}
&\left\|v_{m}(t)\right\|_{\mathbf{V}} \leq \frac{\mu}{4 c_{2}}, \quad \forall t \in[0, T], \forall m, \forall \varepsilon>0 ; \\
& \frac{c}{\kappa}\left\|\theta_{m}(t)\right\|_{W_{\Gamma_{D}}^{1,2}} \leq \frac{\mu}{2}, \quad \forall t \in[0, T], \forall m, \forall \varepsilon>0 .
\end{aligned}
$$

Then, by (91) and (83), we have

$$
\begin{aligned}
&\left\|v_{m}^{\prime}(t)\right\| \leq \text { const, } \quad \forall t \in[0, T], \forall m, \forall \varepsilon>0 ; \\
&\left\|v_{m}^{\prime}\right\|_{L^{2}(0, T ; \mathbf{V})} \leq \text { const, } \quad \forall m, \forall \varepsilon>0 ; \\
&\left\|\theta_{m}^{\prime}(t)\right\| \leq \text { const, } \quad \forall t \in[0, T], \forall m, \forall \varepsilon>0 ; \\
&\left\|\theta_{m}^{\prime}\right\|_{L^{2}\left(0, T ; W_{\Gamma_{D}}^{1,2}\right)} \leq \text { const } \quad \forall m, \forall \varepsilon>0 .
\end{aligned}
$$

By (111),

$$
\int_{0}^{T} \Phi_{\varepsilon}\left(v_{m}(t)\right) \mathrm{d} t \leq \text { const }, \quad \forall m, \forall \varepsilon>0,
$$

and so by (46) and (47),

$$
\int_{0}^{T}\left\|v_{m}(t)-J_{\varepsilon}\left(v_{m}(t)\right)\right\|_{\mathbf{V}}^{2} \mathrm{~d} t \leq c \mathcal{\varepsilon}, \quad \forall m, \forall \varepsilon>0 .
$$

4.2. Existence and Uniqueness of a Solution. Let us prove the existence of a solution. Owing to (111) and (112), we can extract subsequences, which are denoted with the subindex as before, such that

$$
\begin{aligned}
& v_{m} \longrightarrow v \quad \text { in } C([0, T] ; \mathbf{V}), \\
& v_{m}^{\prime} \rightarrow v^{\prime} \quad \text { in } L^{2}(0, T ; \mathbf{V}), \\
& v_{m}^{\prime} \stackrel{*}{\rightarrow} v^{\prime} \quad \text { in } L^{\infty}(0, T ; H), \\
& \theta_{m} \longrightarrow \theta \quad \text { in } C\left([0, T] ; W_{\Gamma_{D}}^{1,2}\right), \\
& \theta_{m}^{\prime} \rightarrow \theta^{\prime} \quad \text { in } L^{2}\left(0, T ; W_{\Gamma_{D}}^{1,2}\right), \\
& \theta_{m}^{\prime} \stackrel{*}{\rightarrow} \theta^{\prime} \quad \text { in } L^{\infty}\left(0, T ; L^{2}(\Omega)\right),
\end{aligned}
$$

when $m \longrightarrow \infty$ and $\varepsilon \longrightarrow 0$.

Putting $u=\sum_{j=1}^{M} k_{j}(t) u_{j}$, where $k_{j}(t) \in C^{1}[0, T]$ and $M$ is the positive integer, multiply the first equation of (52) by $k_{j}(t)$ and add for $j=1, \ldots, M$. Then, multiply the first equation of (52) by $g_{j m}(t)$ and add for $j=1, \ldots, m$. Substituting the resulting equations, we have

$$
\begin{aligned}
& \left\langle v_{m}^{\prime}(t)+A_{1} v_{m}(t)+B_{1}\left(v_{m}(t), v_{m}(t)\right)\right. \\
& \left.\quad+\nabla \Phi_{\varepsilon}\left(v_{m}\right), u(t)-v_{m}(t)\right\rangle=\left\langle\left(1-\alpha_{0} \theta\right) f+f_{1}, u(t)-v_{m}(t)\right\rangle .
\end{aligned}
$$

Since $\Phi_{\varepsilon}$ is convex, continuous, and Fréchet differentiable, we have

$$
\Phi_{\varepsilon}(u(t))-\Phi_{\varepsilon}\left(v_{m}(t)\right) \geq\left\langle\nabla \Phi_{\varepsilon}\left(v_{m}(t)\right), u-v_{m}(t)\right\rangle .
$$

Taking into account (117), we have from (116)

$$
\begin{aligned}
& \int_{0}^{T}\left\langle v_{m}^{\prime}(t)+A_{1} v_{m}(t)+B_{1}\left(v_{m}(t), v_{m}(t)\right)-\left(1-\alpha_{0} \theta_{m}\right) f\right. \\
& \left.\quad-f_{1}, u(t)-v_{m}(t)\right\rangle \mathrm{d} t \\
& \quad+\int_{0}^{T}\left(\Phi_{\varepsilon}(u(t))-\Phi_{\varepsilon}\left(v_{m}(t)\right)\right) \mathrm{d} t \geq 0 .
\end{aligned}
$$

Since $\Phi(u) \geq \Phi_{\varepsilon}(u)$ and $\Phi\left(J_{\varepsilon} w_{m}(t)\right) \leq \Phi_{\varepsilon}\left(w_{m}(t)\right)$ (see (47)), we have from (118)

$$
\begin{aligned}
& \int_{0}^{T}\left\langle v_{m}^{\prime}(t)+A_{1} v_{m}(t)+B_{1}\left(v_{m}(t), v_{m}(t)\right)-\left(1-\alpha_{0} \theta_{m}\right) f\right. \\
& \left.\quad-f_{1}, u(t)-v_{m}(t) \mathrm{d} t\right\rangle \\
& \quad+\Psi(u \mathrm{~d} t)-\int_{0}^{T} \Phi\left(J_{\varepsilon} v_{m}(t)\right) \mathrm{d} t \geq 0 .
\end{aligned}
$$

By $(114), J_{\varepsilon} v_{m} \longrightarrow v$ in $L^{2}(0, T ; \mathbf{V})$ as $m \longrightarrow \infty$ and $\varepsilon \longrightarrow 0$, and by lower semicontinuity of $\Phi$,

$$
\lim _{m \longrightarrow \infty, \varepsilon \longrightarrow 0} \int_{0}^{T} \Phi\left(J_{\varepsilon} v_{m}(t)\right) \mathrm{d} t \geq \int_{0}^{T} \Phi(v(t)) \mathrm{d} t .
$$

In the routine way, we can prove that

$$
\int_{0}^{T}\left\langle B_{1}\left(v_{m}(t), v_{m}(t)\right), v(t)\right\rangle \mathrm{d} t \longrightarrow \int_{0}^{T}\left\langle B_{1}(v(t), v(t)), v(t)\right\rangle \mathrm{d} t
$$

as $m \longrightarrow \infty$ and $\varepsilon \longrightarrow 0$. 
Since $\quad \lim _{m_{k}} \rightarrow \infty, \varepsilon \rightarrow 0\left\langle A_{1} v_{m}(t), \quad v_{m}(t)\right\rangle=\left\langle A_{1} v(t)\right.$, $v(t)\rangle$, by (120) and (121), we have from (119)

$$
\begin{aligned}
& \int_{0}^{T}\left\langle v^{\prime}(t)+A_{1} v(t)+B_{1}(v(t), v(t))\right. \\
& \left.\quad-\left(1-\alpha_{0} \theta\right) f-f_{1}, u(t)-v(t)\right\rangle \mathrm{d} t+\Psi(u)-\Psi(v) \geq 0 .
\end{aligned}
$$

Since $v_{m}(0)=v_{0}$, by (115), it is obvious that $v(0)=v_{0}$. $B_{1}(v(t), v(t)) \in L^{\infty}\left(0, T ; \mathbf{V}^{*}\right), \quad$ and the set $\left\{u=\sum_{j=1}^{M} k_{j}(t) u_{j} ; k_{j}(t) \in C^{1}[0, T], M\right.$ : positive intiger $\}$ is dense in $L^{4}(0, T ; \mathbf{V})$, and so (122) is valid for all $u \in L^{4}(0, T ; \mathbf{V})$.

$$
\begin{aligned}
& \int_{0}^{T}\left|\left\langle v_{m} \theta_{m}, \nabla \varphi\right\rangle-\langle v \theta, \nabla \varphi\rangle\right| \mathrm{d} t \\
& \quad \leq \int_{0}^{T}\left\|v_{m}-v\right\|_{\mathbf{L}^{\prime}}\left\|\theta_{m}\right\|_{L^{3}}\|\nabla \varphi\|_{\mathbf{L}^{2}} \mathrm{~d} t+\int_{0}^{T}\left\langle v\left(\theta_{m}-\theta\right), \nabla \varphi\right\rangle \mathrm{d} t \\
& \quad \leq\left\|v_{m}-v\right\|_{L^{\infty}(0, T ; \mathbf{V})}\left\|\theta_{m}\right\|_{L^{2}\left(0 ; T ; L^{3}\right)}\|\varphi\|_{L^{2}\left(0, T ; W_{\mathrm{I}_{D}}^{1,2}\right)}+\int_{0}^{T}\left|\left\langle v\left(\theta_{m}-\theta\right), \nabla \varphi\right\rangle\right| \mathrm{d} t .
\end{aligned}
$$

$$
\begin{gathered}
\int_{0}^{T}\left\langle\frac{\partial \theta_{m}}{\partial t}, \varphi+\left(\kappa \nabla \theta_{m}, \nabla \varphi\right)+\left(\beta(x) \theta_{m}, \varphi\right)_{\Gamma_{R}}-\left\langle v_{m} \theta_{m}, \nabla \varphi\right\rangle \mathrm{d} t\right\rangle \\
\quad=\int_{0}^{T}\left\langle g_{1}, \varphi\right\rangle \mathrm{d} t, \quad \varphi \in L^{2}\left(0, T ; W_{\Gamma_{D}}^{1,2}\right) .
\end{gathered}
$$

Easily, we see that

$\left(\kappa \nabla \theta_{m}, \nabla \varphi\right) \longrightarrow(\kappa \nabla \theta, \nabla \varphi), \quad$ for a.e. $t \in[0, T]$.

Also,
Since $\quad v \nabla \varphi \in L^{2}\left(0, T ; L^{6 / 5}(\Omega)\right), \quad$ by $\int_{0}^{T}\left|\left\langle v\left(\theta_{m}-\theta\right), \nabla \varphi\right\rangle\right| \mathrm{d} t \longrightarrow 0$. Thus,

$$
\int_{0}^{T}\left\langle v_{m} \theta_{m}, \nabla \varphi\right\rangle \mathrm{d} t \longrightarrow \int_{0}^{T}\langle v \theta, \nabla \varphi\rangle \mathrm{d} t .
$$

It is easy to prove that

$$
\int_{0}^{T}\left(\beta(x) \theta_{m}, \varphi\right)_{\Gamma_{R}} \mathrm{~d} t \longrightarrow \int_{0}^{T}(\beta(x) \theta, \varphi)_{\Gamma_{R}} \mathrm{~d} t
$$

Therefore, from (123), we have

$$
\int_{0}^{T}\left(\left\langle\frac{\partial \theta}{\partial t}, \varphi\right\rangle+(\kappa \nabla \theta, \nabla \varphi)+(\beta(x) \theta, \varphi)_{\Gamma_{R}}-\langle v \theta, \nabla \varphi\rangle\right) \mathrm{d} t=\int_{0}^{T}\left\langle g_{1}, \varphi\right\rangle \mathrm{d} t .
$$

Since $\theta_{m}(0)=\theta_{0}$, by (115), it is obvious that $\theta(0)=\theta_{0}$. Therefore, we proved the existence of a solution.

Let us prove uniqueness of a solution. Let $\left(v_{1}, \theta_{1}\right)$ and $\left(v_{2}, \theta_{2}\right)$ be the two solutions to Problem I-VI satisfying inequality (111) instead of approximate solutions. Then, taking into account (44) (Remark 4), from (35), we have

$$
\begin{aligned}
& \left\langle v_{1}^{\prime}(t)+A_{1} v_{1}(t)+B_{1}\left(v_{1}(t), v_{1}(t)\right)-\left(1-\alpha_{0} \theta_{1}\right) f-f_{1}, v_{2}(t)-v_{1}(t)\right\rangle+\Phi\left(v_{2}(t)\right)-\Phi\left(v_{1}(t)\right) \geq 0, \\
& \left\langle v_{2}^{\prime}(t)+A_{1} v_{2}(t)+B_{1}\left(v_{2}(t), v_{2}(t)\right)-\left(1-\alpha_{0} \theta_{2}\right) f-f_{1}, v_{1}(t)-v_{2}(t)\right\rangle+\Phi\left(v_{1}(t)\right)-\Phi\left(v_{2}(t)\right) \geq 0,
\end{aligned}
$$

which imply

$$
\begin{aligned}
& \left\langle v_{1}^{\prime}(t)-v_{2}^{\prime}(t), v_{1}(t)-v_{2}(t)\right\rangle+\left\langle A_{1}\left(v_{1}(t)-v_{2}(t)\right), v_{1}(t)-v_{2}(t)\right\rangle \\
& \quad \leq\left|\alpha_{0}\right|\left|\left(\theta_{1}-\theta_{2}\right) f, v_{1}(t)-v_{2}(t)\right|+\left|\left\langle B_{1}\left(v_{1}(t), v_{1}(t)\right)-B_{1}\left(v_{2}(t), v_{2}(t)\right), v_{1}(t)-v_{2}(t)\right\rangle\right| .
\end{aligned}
$$

By virtue of (50) and (51), we have 


$$
\begin{aligned}
& \frac{\mathrm{d}}{\mathrm{d} t}\left(\left\|v_{1}(t)-v_{2}(t)\right\|^{2}\right)+\frac{7 \mu}{2}\left\|v_{1}(t)-v_{2}(t)\right\|_{\mathbf{V}}^{2} \\
& \quad \leq 2 c_{*}\left\|v_{1}(t)-v_{2}(t)\right\|^{2}+\frac{\mu}{2}\left\|v_{1}(t)-v_{2}(t)\right\|_{\mathbf{V}}^{2}+c\|f\|_{\mathbf{L}^{3}}^{2}\left\|\theta_{1}-\theta_{2}\right\|^{2} \\
& \quad+2\left|\left\langle B\left(v_{1}(t)-v_{2}(t), v_{1}(t)\right), v_{1}(t)-v_{2}(t)\right\rangle\right|+2\left|\left\langle B\left(v_{2}(t), v_{1}(t)-v_{2}(t)\right), v_{1}(t)-v_{2}(t)\right\rangle\right| \\
& \quad \leq 2 c_{*}\left\|v_{1}(t)-v_{2}(t)\right\|^{2}+\frac{\mu_{\|}}{2}\left\|v_{1}(t)-v_{2}(t)\right\|_{\mathbf{V}}^{2}+c\|f\|_{\mathbf{L}^{3}}^{2}\left\|\theta_{1}-\theta_{2}\right\|^{2} \\
& \quad+2 c_{2}\left(\left\|v_{1}(t)\right\|_{\mathbf{V}}+\left\|v_{2}(t)\right\|_{\mathbf{V}}\right)\left\|v_{1}(t)-v_{2}(t)\right\|_{\mathbf{V}}^{2},
\end{aligned}
$$

where $c_{2}$ is the one in (51). By (111),

$$
2 c_{2}\left(\left\|v_{1}(t)\right\|_{\mathbf{V}}+\left\|v_{2}(t)\right\|_{\mathbf{V}}\right) \leq \mu
$$

and so we have

$$
\begin{aligned}
& \frac{\mathrm{d}\left\|v_{1}(t)-v_{2}(t)\right\|^{2}}{\mathrm{~d} t}+2 \mu\left\|v_{1}(t)-v_{2}(t)\right\|_{\mathrm{V}}^{2} \\
& \quad \leq 2 c_{*}\left\|v_{1}(t)-v_{2}(t)\right\|^{2}+c\|f\|_{\mathrm{L}^{3}}^{2}\left\|\theta_{1}-\theta_{2}\right\|^{2} .
\end{aligned}
$$

Also, from

$$
\left\langle\frac{\partial \theta_{1}}{\partial t}, \varphi\right\rangle+\left(\kappa \nabla \theta_{1}, \nabla \varphi\right)+\left(\beta(x) \theta_{1}, \varphi\right)_{\Gamma_{R}}-\left\langle v_{1} \theta_{1}, \nabla \varphi_{1}\right\rangle=\left\langle g_{1}, \varphi\right\rangle,
$$

$$
\left\langle\frac{\partial \theta_{2}}{\partial t}, \varphi\right\rangle+\left(\kappa \nabla \theta_{2}, \nabla \varphi\right)+\left(\beta(x) \theta_{2}, \varphi\right)_{\Gamma_{R}}-\left\langle v_{2} \theta_{2}, \nabla \varphi\right\rangle=\left\langle g_{1}, \varphi\right\rangle,
$$

we have

$$
\begin{aligned}
& \left\langle\frac{\partial \theta_{1}-\theta_{2}}{\partial t}, \theta_{1}-\theta_{2}\right\rangle+\kappa\left(\nabla \theta_{1}-\nabla \theta_{2}, \nabla \theta_{1}-\nabla \theta_{2}\right)+\left(\beta(x)\left(\theta_{1}-\theta_{2}\right), \theta_{1}-\theta_{2}\right)_{\Gamma_{R}} \\
& \quad-\left\langle v_{1}\left(\theta_{1}-\theta_{2}\right), \nabla\left(\theta_{1}-\theta_{2}\right)\right\rangle-\left\langle\left(v_{1}-v_{2}\right) \theta_{2}, \nabla\left(\theta_{1}-\theta_{2}\right)\right\rangle=0 .
\end{aligned}
$$

Taking into account $\left\langle v_{1}\left(\theta_{1}-\theta_{2}\right), \nabla\left(\theta_{1}-\theta_{2}\right)\right\rangle=0$ (see (17)), by (80), we have

$$
\begin{aligned}
& \frac{\mathrm{d}}{\mathrm{d} t}\left\|\theta_{1}-\theta_{2}\right\|^{2}+2 \kappa\left\|\nabla \theta_{1}-\nabla \theta_{2}\right\|^{2} \\
& \quad \leq \frac{c}{\kappa}\left\|v_{1}(t)-v_{2}(t)\right\|_{\mathrm{V}}^{2}\left\|\theta_{2}(t)\right\|_{W_{\Gamma_{D}}^{1,2}}^{2}+\kappa\left\|\nabla \theta_{1}-\nabla \theta_{2}\right\|^{2},
\end{aligned}
$$

and so

$$
\frac{\mathrm{d}}{\mathrm{d} t}\left(\left\|\theta_{1}(t)-\theta_{2}(t)\right\|^{2}\right) \leq \frac{c}{\kappa}\left\|v_{1}(t)-v_{2}(t)\right\|_{\mathrm{V}}^{2}\left\|\theta_{2}(t)\right\|_{W_{\Gamma_{D}}^{1,2}}^{2} .
$$

By (111),

$$
\frac{c}{\kappa}\left\|\theta_{2}(t)\right\|_{W_{\Gamma_{D}}^{1,2}}^{2} \leq \frac{\mu}{2}
$$

Therefore, adding (133) and (137), we get

$$
\begin{aligned}
& \frac{\mathrm{d}}{\mathrm{d} t}\left(\left\|v_{1}(t)-v_{2}(t)\right\|^{2}+\left\|\theta_{1}(t)-\theta_{2}(t)\right\|^{2}\right) \\
& \quad \leq\left(2 c_{*}+c\|f\|_{\mathrm{L}^{3}}^{2}\right)\left(\left\|v_{1}(t)-v_{2}(t)\right\|^{2}+\left\|\theta_{1}(t)-\theta_{2}(t)\right\|^{2}\right) .
\end{aligned}
$$

We have from (139)

$$
\begin{aligned}
& \left\|v_{1}(t)-v_{2}(t)\right\|^{2}+\left\|\theta_{1}(t)-\theta_{2}(t)\right\|^{2} \\
& \quad \leq \int_{0}^{t}\left(2 c_{*}+2 c\|f\|_{L^{3}}^{2}\right)\left(\left\|v_{1}(s)-v_{2}(s)\right\|^{2}+\left\|\theta_{1}(s)-\theta_{2}(s)\right\|^{2}\right) \mathrm{d} s,
\end{aligned}
$$

which implies $v_{1}(t)=v_{2}(t)$ and $\theta_{1}(t)=\theta_{2}(t)$ for all $t \in[0, T]$.

\section{Proof of Theorem 2}

5.1. Existence and Estimation of a Solution to an Approximate Problem. We first consider a problem approximating (43). For every $0<\varepsilon<1$, let a functional $\Phi_{\varepsilon}$ be defined by (45). 
Let $\left\{u_{j}, j=1,2, \ldots\right\}$ and $\left\{\varphi_{j}, j=1,2, \ldots\right\}$ be, respectively, bases of the space $\mathbf{V}$ and $W_{\Gamma_{D}}^{1,2}(\Omega)$. Without loss of generality, we assume that $u_{1}=v_{0}$ and $\varphi_{1}=\theta_{0}$ as in [26]. We find a solution $v_{m}=\sum_{j=1}^{m} g_{j m}(t) u_{j}$ and $\theta_{m}=\sum_{j=1}^{m} r_{j m}(t) \varphi_{j}$ to the problem

$$
\left\{\begin{array}{l}
\left\langle\frac{\partial v_{m}}{\partial t}, u_{j}\right\rangle+2\left\langle\mu\left(\theta_{m}\right) \mathscr{E}\left(v_{m}\right), \mathscr{E}\left(u_{j}\right)\right\rangle+\left\langle\operatorname{rot} v_{m} \times v_{m}, u_{j}\right\rangle+2\left(\mu\left(\theta_{m}\right) k(x) v_{m}, u_{j}\right)_{\Gamma_{2}} \\
+2\left(\mu\left(\theta_{m}\right) S \widetilde{v}_{m}, \widetilde{u}_{j}\right)_{\Gamma_{3}}+2\left(\alpha(x) v_{m}, u_{j}\right)_{\Gamma_{5}}+\left(\mu\left(\theta_{m}\right) k(x) v_{m}, u_{j}\right)_{\Gamma_{7}}+\left\langle\nabla \Phi_{\varepsilon}\left(v_{m}(t)\right), u_{j}\right\rangle \\
=\left\langle\left(1-\alpha_{0} \theta_{m}\right) f, u_{j}\right\rangle+\left\langle f_{2}, u_{j}\right\rangle, \\
\left\langle\frac{\partial \theta_{m}}{\partial t}, \varphi_{j}\right\rangle+\left(\kappa\left(\theta_{m}\right) \nabla \theta_{m}, \nabla \varphi_{j}\right)+\left(\beta(x) \theta_{m}, \varphi_{j}\right)_{\Gamma_{R}}-\left\langle v_{m} \theta_{m}, \nabla \varphi_{j}\right\rangle=\left\langle g_{2}, \varphi_{j}\right\rangle, \\
v_{m}(0)=v_{0}, \\
\theta_{m}(0)=\theta_{0},
\end{array}\right.
$$

which gives us a system for $g_{j m}(t)$ and $r_{j m}(t), j=1-m$. The solutions to (141) depend on $\varepsilon$, but for convenience of notation, here and in what follows, we use subindex $m$. For $t_{m}$, there exist absolute continuous functions $g_{j m}(t)$ and $r_{j m}(t)$ on $\left[0, t_{m}\right)$. If $\left\|v_{m}(t)\right\|$ and $\left\|\theta_{m}(t)\right\|$ are bounded and $v_{m}(t)$ and $\theta_{m}(t)$ are integrable, then $g_{j m}(t)$ and $r_{j m}(t)$ are prolonged over $t_{m}$. We will find estimates (157) in the following, by which we see that $t_{m}=T$.

Multiplying the first and the second equation of (141), respectively, by $g_{j m}(t)$ and $\varphi_{j m}(t)$ and adding for $i=1, \ldots, m$, we get

$$
\left\{\begin{array}{l}
\left\langle\frac{\partial v_{m}}{\partial t}, v_{m}\right\rangle+2\left\langle\mu\left(\theta_{m}\right) \mathscr{E}\left(v_{m}\right), \mathscr{E}\left(v_{m}\right)\right\rangle+\left\langle\operatorname{rot} v_{m} \times v_{m}, v_{m}\right\rangle+2\left(\mu\left(\theta_{m}\right) k(x) v_{m}, v_{m}\right)_{\Gamma_{2}} \\
+2\left(\mu\left(\theta_{m}\right) S \widetilde{v}_{m}, \widetilde{v}_{m}\right)_{\Gamma_{3}}+2\left(\alpha(x, t) v_{m}, v_{m}\right)_{\Gamma_{5}}+\left(\mu\left(\theta_{m}\right) k(x) v_{m}, v_{m}\right)_{\Gamma_{7}} \\
+\left\langle\nabla \Phi_{\varepsilon}\left(v_{m}(t)\right), v_{m}\right\rangle+\left\langle\alpha_{0} \theta_{m} f, v_{m}\right\rangle=\left\langle f, v_{m}\right\rangle+\left\langle f_{2}, v_{m}\right\rangle, \\
\left\langle\frac{\partial \theta_{m}}{\partial t}, \theta_{m}\right\rangle+\left(\kappa\left(\theta_{m}\right) \nabla \theta_{m}, \nabla \theta_{m}\right)+\left(\beta(x) \theta_{m}, \theta_{m}\right)_{\Gamma_{R}}-\left\langle v_{m} \theta_{m}, \nabla \theta_{m}\right\rangle=\left\langle g_{2}, \theta_{m}\right\rangle, \\
v_{m}(0)=v_{0} \\
\theta_{m}(0)=\theta_{0} .
\end{array}\right.
$$

Let us estimate terms on the left-hand side above. It is easy to see

$$
2\left\langle\mu\left(\theta_{m}\right) \mathscr{E}\left(v_{m}\right), \mathscr{E}\left(v_{m}\right)\right\rangle \mathrm{d} t \geq 2 \mu_{0}\left\|v_{m}\right\|_{\mathrm{V}}^{2} .
$$

By the fact that $\Gamma_{2 j}, \Gamma_{3 j}, \Gamma_{7 j}$ are in $C^{2.1}\left(\Gamma_{i j}\right)$ and Assumption 2, there exists a constant $M$ such that

$$
\|S(x)\|_{\infty},\|k(x)\|_{\infty},\|\alpha\|_{L^{\infty}\left(\Gamma_{5}\right)} \leq M .
$$

Thus,

$$
\begin{aligned}
& \mid\left(2\left(\mu\left(\theta_{m}\right) k(x) v, v\right)_{\Gamma_{2}}+2\left(\mu\left(\theta_{m}\right) S \widetilde{v}_{m}, \widetilde{v}_{m}\right)_{\Gamma_{3}}+2\left(\alpha(x) v_{m}, v_{m}\right)_{\Gamma_{5}}\right. \\
& \left.\quad+\left(\mu\left(\theta_{m}\right) k(x) v_{m}, v_{m}\right)_{\Gamma_{7}}\right) \mid \\
& \quad \leq \frac{\mu_{0}}{2}\left\|v_{m}\right\|_{\mathrm{V}}^{2}+k_{11}\left\|v_{m}\right\|^{2},
\end{aligned}
$$

(cf. Theorem 1.5.1.10 of [28]). Obviously,

$$
\left\langle\operatorname{rot} v_{m} \times v_{m}, v_{m}\right\rangle=0 .
$$


Since $\Phi_{\varepsilon}$ is convex, continuous and Fréchet differentiable, we have

$\Phi_{\varepsilon}(y)-\Phi_{\varepsilon}(x) \geq\left\langle\nabla \Phi_{\varepsilon}(x), y-x\right\rangle, \quad \forall x, y \in \mathbf{V}$.

Thus,

$\left\langle\nabla \Phi_{\varepsilon}\left(v_{m}(t)\right), 0_{\mathbf{V}}-v_{m}(t)\right\rangle \leq \Phi_{\varepsilon}\left(0_{\mathbf{V}}\right)-\Phi_{\varepsilon}\left(v_{m}(t)\right) \leq-\Phi_{\varepsilon}\left(v_{m}(t)\right)$,

$\left\langle\nabla \Phi_{\varepsilon}\left(v_{m}(t)\right), v_{m}(t)\right\rangle \geq \Phi_{\varepsilon}\left(v_{m}(t)\right)$.

Also, by the Hölder inequality, we have

$$
\left|\left\langle\alpha_{0} \theta_{m} f, v_{m}\right\rangle \mathrm{d} t\right| \leq k_{12}\left\|v_{m}(t)\right\|^{2}+\frac{\kappa_{0}}{4}\left\|\theta_{m}(t)\right\|_{W_{\Gamma_{D}}^{1,2}}^{2}
$$

where $k_{12}=c\left|\alpha_{0}\right|\|f\|_{L^{\infty}\left(0, T ; \mathbf{L}^{3}\right)}^{2}$.

$$
\left|\left\langle f, w_{m}\right\rangle+\left\langle f_{2}, w_{m}\right\rangle\right| \leq \frac{\mu_{0}}{2}\left\|w_{m}\right\|_{\mathbf{V}}^{2}+c\left(\|f\|_{\mathbf{L}^{3}}^{2}+\left\|f_{2}\right\|_{\mathbf{v}^{*}}^{2}\right) .
$$

Also, we have

$$
\left\langle\kappa\left(\theta_{m}\right) \nabla \theta_{m}, \nabla \theta_{m}\right\rangle \geq \kappa_{0}\left\|\theta_{m}\right\|_{W_{\Gamma_{D}}^{1,2}}^{2}, \quad\left(\beta(x) \theta_{m}, \theta_{m}\right)_{\Gamma_{R}} \geq 0 .
$$

By (17), we have

$$
\begin{aligned}
\left\langle w_{m} \theta_{m}, \nabla \theta_{m}\right\rangle & =0 . \\
\left|\left\langle g_{2}, \theta_{m}\right\rangle\right| & \leq \frac{\kappa_{0}}{4}\left\|\theta_{m}(t)\right\|_{W_{\Gamma_{D}}^{1,2}}^{2}+c\left\|g_{2}\right\|_{\left(W_{\Gamma_{D}}^{1,2}\right)^{*}}^{2} .
\end{aligned}
$$

Taking

$$
k_{1}=k_{11}+k_{12}
$$

by (143)-(154), we have from (142)

$$
\begin{aligned}
& \frac{\mathrm{d}}{\mathrm{d} t}\left\|v_{m}(t)\right\|^{2}+\frac{\mathrm{d}}{\mathrm{d} t}\left\|\theta_{m}(t)\right\|^{2}+2 \mu_{0}\left\|v_{m}\right\|_{\mathbf{V}}^{2}+\kappa_{0}\left\|\theta_{m}\right\|_{W_{\Gamma_{D}}^{1,2}}^{2}+\Phi_{\varepsilon}\left(v_{m}(t)\right) \\
& \quad \leq c\left(\|f(t)\|_{\mathbf{L}^{3}}^{2}+\left\|f_{2}(t)\right\|_{\mathbf{v}^{*}}^{2}+\left\|g_{2}(t)\right\|_{\left(W_{\Gamma_{D}}^{1,2}\right)^{*}}^{2}\right)+2 k_{1}\left\|v_{m}(t)\right\|^{2} .
\end{aligned}
$$

Applying the Gronwall inequality, we have from (156)

$$
\begin{aligned}
\left\|v_{m}(t)\right\|^{2}+\left\|\theta_{m}(t)\right\|^{2} & \leq\left[\left\|v_{0}\right\|^{2}+\left\|\theta_{0}\right\|^{2}+\int_{0}^{t}\left(\|f(s)\|_{\mathbf{L}^{3}}^{2}+\left\|f_{2}(s)\right\|_{\mathbf{v}^{*}}^{2}+\left\|g_{2}(s)\right\|_{\left(W_{\Gamma_{D}}^{1,2}\right)}^{2}\right) \mathrm{d} s\right] e^{2 k_{1} t}, \\
\left\|v_{m}\right\|_{L^{2}(0, T ; \mathbf{V})}^{2}+\left\|\theta_{m}\right\|_{L^{2}\left(0, t ; W_{\Gamma_{D}}^{1,2}\right.}^{2} & \leq c\left(\left\|v_{0}\right\|^{2}+\left\|\theta_{0}\right\|^{2}+\int_{0}^{T}\left(\|f(t)\|_{\mathbf{L}^{3}}^{2}+\left\|f_{2}(t)\right\|_{\mathbf{v}^{*}}^{2}+\left\|g_{2}(t)\right\|_{\left(W_{\Gamma_{D}}^{1,2}\right)^{*}}^{2}\right)\right) \mathrm{d} t \\
\int_{0}^{T} \Phi_{\varepsilon}\left(v_{m}(t)\right) \mathrm{d} t & \leq c\left(\left\|v_{0}\right\|^{2}+\left\|\theta_{0}\right\|^{2}+\int_{0}^{T}\left(\|f(t)\|_{\mathbf{L}^{3}}^{2}+\left\|f_{2}(t)\right\|_{\mathbf{v}^{*}}^{2}+\left\|g_{2}(t)\right\|_{\left(W_{\Gamma_{D}}^{1,2}\right)^{*}}^{2}\right) \mathrm{d} t\right) .
\end{aligned}
$$

Note that $c$ in (157) depends on $T$ and (via $\left.k_{12}\right) f$ but independent of $m$ and $\varepsilon$.

By (46) and (29) and the third inequality of (157), we have

$$
\int_{0}^{T}\left\|v_{m}(t)-J_{\varepsilon}\left(v_{m}(t)\right)\right\|_{\mathbf{V}}^{2} \mathrm{~d} t \leq c \mathcal{E}
$$

$$
\begin{gathered}
\frac{1}{2} \frac{\mathrm{d}\left\|v_{m}(t)-v_{m}(s)\right\|^{2}}{\mathrm{~d} t}+\left\langle A_{2}\left(\theta_{m}\right) v_{m}(t)+B_{2}\left(v_{m}(t), v_{m}(t)\right)+\alpha_{0} \theta_{m}(t) f(t)-f(t)-f_{2}(t), v_{m}(t)-v_{m}(s)\right\rangle \\
\quad=\left\langle\nabla \Phi_{\varepsilon}\left(v_{m}(t)\right), v_{m}(s)-v_{m}(t)\right\rangle \leq \Phi_{\varepsilon}\left(v_{m}(s)\right)-\Phi_{\varepsilon}\left(v_{m}(s)\right) \leq \Phi_{\varepsilon}\left(v_{m}(s)\right),
\end{gathered}
$$

where the operators $A_{2}\left(\theta_{m}\right), B_{2}$ are the ones in (42). By (145) and (144), we have

$$
\begin{gathered}
\left\langle A_{2}\left(\theta_{m}\right) v_{m}(t), v_{m}(t)\right\rangle \geq \frac{3 \mu_{0}}{2}\left\|v_{m}(t)\right\|_{\mathbf{V}}^{2}-k_{11}\left\|v_{m}(t)\right\|^{2}, \\
\left|\left\langle A_{2}\left(\theta_{m}\right) v_{m}(t), v_{m}(s)\right\rangle\right| \leq c\left\|v_{m}(t)\right\|_{\mathbf{V}}\left\|v_{m}(s)\right\|_{\mathbf{V}},
\end{gathered}
$$


where $k_{11}$ is the one in (145). Taking into account (160) and the fact that $\left\langle B_{2}\left(v_{m}(t), v_{m}(t)\right), v_{m}(t)\right\rangle=0$, we have from (159)

$$
\begin{aligned}
\frac{1}{2} \frac{\mathrm{d}\left\|v_{m}(t)-v_{m}(s)\right\|^{2}}{\mathrm{~d} t} \leq & \Phi_{\varepsilon}\left(v_{m}(s)\right)+\left\langle A_{2}\left(\theta_{m}\right) v_{m}(t), v_{m}(s)\right\rangle+\left\langle B_{2}\left(v_{m}(t), v_{m}(t)\right), v_{m}(s)\right\rangle \\
& +\left\langle-\alpha_{0} \theta_{m}(t) f(t)+f(t)+f_{2}(t), v_{m}(t)-v_{m}(s)\right\rangle+k_{11}\left\|v_{m}(t)\right\|^{2}
\end{aligned}
$$

Let us integrate every term of (161) first with respect to $t$ from $s$ to $s+h$ and then with respect to $s$ from 0 to $T$, where $v_{m}(t)=0$ when $t \in(T, T+h)$.

$$
\int_{0}^{T} \int_{s}^{s+h} \frac{\mathrm{d}\left\|v_{m}(t)-v_{m}(s) \mid\right\|^{2}}{\mathrm{~d} t} \mathrm{~d} t \mathrm{~d} s=\int_{0}^{T}\left\|v_{m}(s+h)-v_{m}(s)\right\|^{2} \mathrm{~d} s .
$$

By the third inequality of (157),

$$
\int_{0}^{T} \int_{s}^{s+h} \Phi_{\varepsilon}\left(v_{m}(s)\right) \mathrm{d} t \mathrm{~d} s \leq h \int_{0}^{T} \Phi_{\varepsilon}\left(v_{m}(s)\right) \mathrm{d} s \leq c_{1} h .
$$

By (157) and (161), we have

$$
\begin{aligned}
& \quad\left|\int_{0}^{T} \int_{s}^{s+h}\left\langle A_{2}\left(\theta_{m}\right) v_{m}(t), v_{m}(s)\right\rangle \mathrm{d} t \mathrm{~d} s\right| \\
& \quad \leq c \int_{0}^{T}\left\|v_{m}(s)\right\|_{\mathbf{V}} \int_{s}^{s+h}\left\|v_{m}(t)\right\|_{\mathbf{V}} \mathrm{d} t \mathrm{~d} s \\
& \quad \leq c \int_{0}^{T}\left\|v_{m}(s)\right\|_{\mathbf{V}}\left(\sqrt{h}\left\|v_{m}\right\|_{L^{2}(0, T ; \mathbf{V})}\right) \mathrm{d} s \leq c_{2} \sqrt{h}
\end{aligned}
$$

Since $\|w\|_{\mathbf{L}^{3}} \leq K\|w \mid\|_{\mathbf{L}^{2}}^{1 / 2}\|w\|_{\mathbf{L}^{6}}^{1 / 2}$

$$
\begin{aligned}
\left|\left\langle B_{2}(v, w), z\right\rangle\right| & =|\langle\operatorname{rot} v \times w, z\rangle| \\
& \leq K\|\operatorname{rot} v \mid\|_{\mathbf{L}^{2}}\|w\|_{\mathbf{L}^{3}}\|z\|_{\mathbf{L}^{6}} \\
& \leq K\|v\|_{\mathbf{V}}\|w\|^{1 / 2}\|w\|_{\mathbf{V}}^{1 / 2}\|z\|_{\mathbf{V}},
\end{aligned}
$$

and so by (157), we have

$$
\begin{aligned}
& \left|\int_{0}^{T} \int_{s}^{s+h}\left\langle B_{2}\left(v_{m}(t), v_{m}(t)\right) v_{m}(s)\right\rangle\right| \mathrm{d} t \mathrm{~d} s \\
& \quad \leq K \int_{0}^{T} \int_{s}^{s+h}\left\|v_{m}(t)\right\|_{\mathrm{V}}^{3 / 2}\left\|v_{m}(t)\right\|^{1 / 2}\left\|v_{m}(s)\right\|_{\mathrm{V}} \mathrm{d} t \mathrm{~d} s \\
& \quad \leq K \int_{0}^{T}\left\|v_{m}(s)\right\|_{\mathrm{V}}\left(\int_{s}^{s+h}\left\|v_{m}(t)\right\|_{\mathrm{V}}^{2} \mathrm{~d} t\right)^{3 / 4}\left(\int_{s}^{s+h}\left\|v_{m}(t)\right\|^{2} \mathrm{~d} t\right)^{1 / 4} \mathrm{~d} s \leq c_{3} h^{1 / 4} .
\end{aligned}
$$

Also, by (157), we have

$$
\begin{gathered}
\left|\int_{0}^{T} \int_{s}^{s+h}\left\langle\left(f+f_{2}\right)(t), v_{m}(t)\right\rangle \mathrm{d} t \mathrm{~d} s\right| \leq \int_{0}^{T}\left|\left\langle\left(f+f_{2}\right)(t), v_{m}(t)\right\rangle\right|\left(\int_{t-h}^{t} \mathrm{~d} s\right) \mathrm{d} t \leq c_{4} h, \\
\left|\int_{0}^{T} \int_{s}^{s+h}\left\langle\left(f+f_{2}\right)(t),-v_{m}(s)\right\rangle \mathrm{d} t \mathrm{~d} s\right| \leq K \int_{0}^{T}\left\|v_{m}(s)\right\|_{\mathbf{V}} \int_{s}^{s+h}\left\|\left(f+f_{2}\right)(t)\right\| \|_{\mathbf{V}^{*}} \mathrm{~d} t \mathrm{~d} s \leq c_{5} \sqrt{h} .
\end{gathered}
$$

In the same way, we get

$$
\begin{gathered}
\left|\int_{0}^{T} \int_{s}^{s+h}\left\langle\alpha_{0} \theta_{m} f, v_{m}(t)-v_{m}(s)\right\rangle \mathrm{d} t \mathrm{~d} s\right| \leq c_{6} h+c_{7} \sqrt{h}, \\
\left|\int_{0}^{T} \int_{s}^{s+h} k_{11}\right|\left|v_{m}(t) \|^{2} \mathrm{~d} t \mathrm{~d} s\right| \leq c_{8} h .
\end{gathered}
$$

Note that constants $c_{i}, i=1-8$, are independent of $m, \varepsilon$. By virtue of (162)-(168), uniformly with respect to $m, \varepsilon$,

$$
\int_{0}^{T}\left\|v_{m}(s+h)-v_{m}(s)\right\|^{2} \mathrm{~d} s \leq O\left(h^{1 / 4}\right)
$$

and the set $\left\{v_{m}\right\}$ is relatively compact in $L^{2}\left(0, T ; \mathbf{W}^{9 / 10,2}(\Omega)\right)$ (see Theorem 5 of [29]). Also, we have

$$
\begin{aligned}
\left|\left(\kappa\left(\theta_{m}\right) \nabla \theta_{m}, \nabla \varphi\right)\right| & \leq \kappa_{1}\left\|\nabla \theta_{m}\right\|_{L^{2}}\|\varphi\|_{W_{\Gamma_{D}}^{1,2}(\Omega)}, \\
\left|\left(\beta(x) \theta_{m}, \varphi\right)_{\Gamma_{R}}\right| & \leq c\left\|\theta_{m}\right\|_{W_{\Gamma_{D}}^{1,2}(\Omega)}\|\varphi\|_{W_{\Gamma_{D}}^{1,2}(\Omega)}, \\
\left|\left\langle v_{m} \theta_{m}, \nabla \varphi\right\rangle\right| & \leq c\left\|v_{m}\right\|_{\mathrm{V}}\left\|\theta_{m}\right\|_{L^{3}}\|\varphi\|_{W_{\Gamma_{D}}^{1,2}(\Omega)} .
\end{aligned}
$$

By (170), from the second equation of (141), we have 


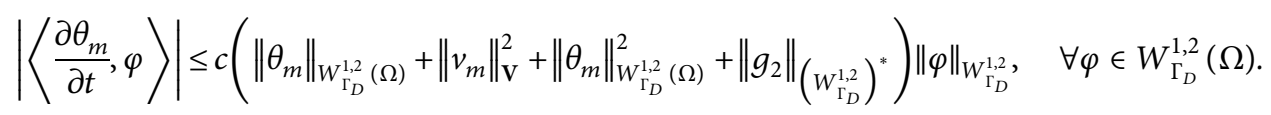

Hence, by (157), we know that

$$
\theta_{m}^{\prime} \in L^{1}\left(0, T ;\left(W_{\Gamma_{D}}^{1, \tau}\right)^{*}\right), \quad\left\|\theta_{m}^{\prime}\right\|_{L^{1}\left(0, T ;\left(W_{\Gamma_{D}}^{1, \tau}\right)^{*}\right)} \leq c,
$$

where $c$ is independent of $m, \varepsilon$. Thus, the set $\left\{\theta_{\varepsilon}\right\}$ is relatively compact in $L^{2}\left(0, T ; W^{9 / 10,2}(\Omega)\right)$ (see Corollary 5 of [29]). as $m \longrightarrow \infty$ and $\varepsilon \longrightarrow 0$.

On the contrary, putting $u=\sum_{j=1}^{M} k_{j}(t) u_{j}$, where $k_{j}(t) \in C^{1}[0, T]$ and is a positive integer, multiply the first equation of (141) by $k_{j}(t)$ and add for $j=1, \ldots, M$. Then, multiply the first equation of (141) by $g_{j m}(t)$ and add for $j=1, \ldots, m$. Substituting the resulting equations, we have

5.2. Existence of a Solution. We can extract subsequences, which are denoted as before, such that

$$
\begin{array}{ll}
v_{m} \rightarrow v & \text { in } L^{2}(0, T ; \mathbf{V}), \\
v_{m} \stackrel{*}{\rightarrow} v & \text { in } L^{\infty}(0, T ; H), \\
v_{m} \longrightarrow v & \text { in } L^{2}\left(0, T ; \mathbf{W}^{9 / 10,2}(\Omega)\right), \\
\theta_{m} \rightarrow \theta & \text { in } L^{2}\left(0, T ; W_{D}^{1,2}(\Omega)\right), \\
\theta_{m} \stackrel{*}{\rightarrow} \theta & \text { in } L^{\infty}\left(0, T ; L^{2}(\Omega)\right), \\
\theta_{m} \longrightarrow \theta & \text { in } L^{2}\left(0, T ; W^{9 / 10,2}(\Omega)\right),
\end{array}
$$

$$
\begin{aligned}
& \int_{0}^{T}\left\langle\frac{\partial v_{m}}{\partial t}+A_{2}\left(\theta_{m}\right) v_{m}(t)+B_{2}\left(v_{m}(t), v_{m}(t)\right)+\nabla \Phi_{\varepsilon}\left(v_{m}(t)\right), u(t)-v_{m}(t)\right\rangle \mathrm{d} t \\
& \quad=\int_{0}^{T}\left\langle-\alpha_{0} \theta_{m} f+f+f_{2}, u(t)-v_{m}(t)\right\rangle d t .
\end{aligned}
$$

$$
\begin{aligned}
& \text { Since } \\
& \begin{array}{l}
\int_{0}^{T}\left\langle v_{m}^{\prime}(t), u(t)-v_{m}(t)\right\rangle \mathrm{d} t=\int_{0}^{T}\left\langle u \prime(t), u(t)-v_{m}(t)\right\rangle \mathrm{d} t \\
\quad-\frac{1}{2}\left\|v_{m}(T)-u(T)\right\|^{2}+\frac{1}{2}\left\|v_{m}(0)-u(0)\right\|^{2},
\end{array}
\end{aligned}
$$

taking into account (147), we have from (174)

$$
\begin{aligned}
& \int_{0}^{T}\left\langle u^{\prime}(t)+A_{2}\left(\theta_{m}\right) v_{m}(t)+B_{2}\left(v_{m}(t), v_{m}(t)\right), u(t)-v_{m}(t)\right\rangle \mathrm{d} t \\
& -\int_{0}^{T}\left\langle-\alpha_{0} \theta_{m} f+f+f_{2}, u(t)-v_{m}(t)\right\rangle \mathrm{d} t \\
& \quad+\int_{0}^{T}\left(\Phi_{\varepsilon}(u(t))-\Phi_{\varepsilon}\left(v_{m}(t)\right)\right) \mathrm{d} t \geq-\frac{1}{2}\left\|v_{m}(0)-u(0)\right\|^{2} .
\end{aligned}
$$

Since $\Phi_{\varepsilon}(u) \leq \Phi(u)$ and $\Phi\left(J_{\varepsilon} v_{m}(t)\right) \leq \Phi_{\varepsilon}\left(v_{m}(t)\right)$ (cf. (47)), we have from (176)

$$
\begin{aligned}
& \int_{0}^{T}\left\langle u^{\prime}(t)+A_{2}\left(\theta_{m}\right) v_{m}(t)+B_{2}\left(v_{m}(t), v_{m}(t)\right), u(t)-v_{m}(t)\right\rangle \mathrm{d} t \\
& -\int_{0}^{T}\left\langle-\alpha_{0} \theta_{m} f+f+f_{2}, u(t)-v_{m}(t)\right\rangle \mathrm{d} t+\Psi(u) \\
& \quad-\int_{0}^{T} \Phi\left(J_{\varepsilon} v_{m}(t)\right) \mathrm{d} t \geq-\frac{1}{2}\left\|v_{m}(0)-u(0)\right\|^{2} .
\end{aligned}
$$

By (173) and Corollary Appendix B.2 of [1], taking a subsequence if necessary, we have

$$
\begin{aligned}
& \int_{0}^{T}\left\langle A_{2}\left(\theta_{m}\right) v_{m}(t), u(t)\right\rangle \mathrm{d} t \equiv \int_{0}^{T} a_{02}\left(\theta_{m}(t) ; v_{m}(t), u(t)\right) \mathrm{d} t \\
& \longrightarrow \int_{0}^{T}\left\langle A_{2}(\theta) v(t), u(t)\right\rangle \mathrm{d} t .
\end{aligned}
$$

Owing to (173),

$$
v_{m} \longrightarrow v \operatorname{in} L^{2}\left(0, T ; \mathbf{L}^{2}(\partial \Omega)\right) .
$$

Thus, taking a subsequence if necessary, we have (see Lemma Appendix B.1 of [1]) 


$$
\begin{aligned}
& 2\left(\mu\left(\theta_{m}\right) k(x) v_{m}, v_{m}\right)_{\Gamma_{2}}+2\left(\mu\left(\theta_{m}\right) S \widetilde{v}_{m}, \widetilde{v}_{m}\right)_{\Gamma_{3}} \\
& \quad+2\left(\alpha(x) v_{m}, v_{m}\right)_{\Gamma_{5}}+\left(\mu\left(\theta_{m}\right) k(x) v_{m}, v_{m}\right)_{\Gamma_{7}} \longrightarrow \\
& \quad+2(\mu(\theta) k(x) v, v)_{\Gamma_{2}}+2(\mu(\theta) S \widetilde{v}, \widetilde{v})_{\Gamma_{3}}+2(\alpha(x) v, v)_{\Gamma_{5}} \\
& \quad+(\mu(\theta) k(x) v, v)_{\Gamma_{7}} .
\end{aligned}
$$

$$
\liminf 2\left(\mu\left(\theta_{m}\right) \mathscr{E}\left(v_{m}\right), \mathscr{E}\left(v_{m}\right)\right) \geq 2(\mu(\theta) \mathscr{E}(v), \mathscr{E}(v))
$$

(see Corollary Appendix B.3 of [1]), we have

$$
\lim \inf \left\langle A_{2}\left(\theta_{m}\right) v_{m}(t), v_{m}(t)\right\rangle \geq\left\langle A_{2}(\theta) v(t), v(t)\right\rangle .
$$

By (178) and (182), we have

Therefore, taking into account

$$
\liminf _{\substack{m \longrightarrow \infty \\ \varepsilon \longrightarrow 0}} \int_{0}^{T}\left\langle A_{2}\left(\theta_{m}\right) v_{m}(t), u(t)-v_{m}(t)\right\rangle \mathrm{d} t \leq \int_{0}^{T}\left\langle A_{2}(\theta) v(t), u(t)-v(t)\right\rangle \mathrm{d} t .
$$

By (158) and (173), $J_{\varepsilon}\left(v_{m}\right) \rightarrow v$ in $L^{2}(0, T ; \mathbf{V})$ as $m \longrightarrow \infty$ and $\varepsilon \longrightarrow 0$. Since the functional $\Phi: \mathbf{V} \longrightarrow \overline{\mathbb{R}}$ is lower weak semicontinuous, we have

$$
\liminf _{\substack{i \\ \varepsilon \longrightarrow 0}} \int_{0}^{T} \Phi\left(J_{\varepsilon} v_{m}(t)\right) \mathrm{d} t \geq \int_{0}^{T} \Phi(v(t)) \mathrm{d} t \equiv \Psi(v)
$$

In a rather routine way, we can prove that

$$
\int_{0}^{T}\left\langle B_{2}\left(v_{m}(t), v_{m}(t)\right), u(t)\right\rangle \mathrm{d} t \longrightarrow \int_{0}^{T}\left\langle B_{2}(v(t), v(t)), u(t)\right\rangle \mathrm{d} t
$$

as $m \longrightarrow \infty, \varepsilon \longrightarrow 0$. For convenience of readers, we give a proof in the following:

$$
\begin{aligned}
& \int_{0}^{T}\left(\left\langle B_{2}\left(v_{m}(t), v_{m}(t)\right), u(t)\right\rangle-\left\langle B_{2}(v(t), v(t)), u(t)\right\rangle\right) \mathrm{d} t \\
& \quad=\int_{0}^{T}\left\langle B_{2}\left(v_{m}(t), v_{m}(t)-v(t)\right), u(t)\right\rangle \mathrm{d} t+\int_{0}^{T}\left\langle B_{2}\left(v_{m}(t)-v(t), v(t)\right), u(t)\right\rangle \mathrm{d} t \equiv I_{1}+I_{2} .
\end{aligned}
$$

By (165), the Hölder inequality with exponents 2, 4, 4, and (173), we have

$$
\begin{aligned}
& \left|I_{1}\right| \leq K \sup _{t \in[0, T]}\|u(t)\|_{\mathbf{H}^{1}} \int_{0}^{T}\left\|v_{m}(t)\right\|_{\mathbf{H}^{1}}\left\|v_{m}(t)-v(t)\right\|_{\mathbf{H}^{1}}^{1 / 2}\left\|v_{m}(t)-v(t)\right\|^{1 / 2} \mathrm{~d} t \\
& \quad \leq K \sup _{t \in[0, T]}\|u(t)\|_{\mathbf{H}^{1}}\left\|v_{m}\right\|_{L^{2}\left(0, T ; \mathbf{H}^{1}\right)}\left\|v_{m}-v\right\|_{L^{2}\left(0, T ; \mathbf{H}^{1}\right)}^{1 / 2}\left\|v_{m}-v\right\|_{L^{2}\left(0, T ; \mathbf{L}^{2}\right)}^{1 / 2} \longrightarrow 0 .
\end{aligned}
$$

By (165),

$$
\begin{aligned}
& \left|\int_{0}^{T}\left\langle B_{2}(z(t), v(t)), u(t)\right\rangle \mathrm{d} t\right| \\
& \quad \leq K \sup _{t \in[0, T]}\|u(t)\|_{\mathbf{H}^{1}}\|z(t)\|_{L^{2}\left(0, T ; \mathbf{H}^{1}\right)}\|v\|_{L^{2}\left(0, T ; \mathbf{H}^{1}\right)}^{1 / 2}\|v\|_{L^{2}\left(0, T ; \mathbf{L}^{2}\right)}^{1 / 2} \\
& \quad \leq K_{1}\|z(t)\|_{L^{2}\left(0, T ; \mathbf{H}^{1}\right)},
\end{aligned}
$$

which means that the mapping

$$
z(t) \in L^{2}(0, T ; \mathbf{V}) \longrightarrow \int_{0}^{T}\left\langle B_{2}(z(t), v(t)), u(t)\right\rangle \mathrm{d} t,
$$

is continuous and linear on $L^{2}(0, T ; \mathbf{V})$, that is, there exists $f \in L^{2}(0, T ; \mathbf{V})^{*}$ such that

$$
\int_{0}^{T}\left\langle B_{2}(z(t), v(t)), u(t)\right\rangle \mathrm{d} t=\int_{0}^{T}\langle z(t), f(t)\rangle \mathrm{d} t .
$$

Thus, by (173),

$$
\left|I_{2}\right| \longrightarrow 0, \quad \text { as } m \longrightarrow \infty, \varepsilon \longrightarrow 0 .
$$

By (186)-(191), we get (185).

Let us prove that 


$$
\begin{gathered}
\int_{0}^{T}\left\langle\alpha_{0} \theta_{m} f, u(t)-v_{m}(t)\right\rangle \mathrm{d} t \longrightarrow \int_{0}^{T}\left\langle\alpha_{0} \theta f, u(t)-v(t)\right\rangle \\
\mathrm{d} t \longrightarrow 0, \quad \text { as } m \longrightarrow \infty, \varepsilon \longrightarrow 0
\end{gathered}
$$

By (173), we have

$$
\begin{aligned}
& \int_{0}^{T}\left\langle\left(\alpha_{0} \theta_{m}-\alpha_{0} \theta\right) f, u(t)\right\rangle \mathrm{d} t \longrightarrow 0, \\
& \int_{0}^{T}\left(\left\langle\alpha_{0} \theta_{m} f, v_{m}(t)\right\rangle-\left\langle\alpha_{0} \theta f, v(t)\right\rangle\right) \mathrm{d} t \\
&=\int_{0}^{T}\left\langle\alpha_{0}\left(\theta_{m}-\theta\right) f, v_{m}(t)\right\rangle \mathrm{d} t \\
&+\int_{0}^{T}\left\langle\alpha_{0} \theta f, v_{m}(t)-v(t)\right\rangle \mathrm{d} t \longrightarrow 0 .
\end{aligned}
$$

By (193) and (194), we get (192).

Therefore, by (183)-(185) and (192), from (177), we have

$$
\begin{aligned}
& \int_{0}^{T}\left\langle u^{\prime}(t)+A_{2}(\theta) v(t)+B_{2}(v(t), v(t))-\left(1-\alpha_{0} \theta\right) f\right. \\
& \left.\quad-f_{2}, u(t)-v(t)\right\rangle \mathrm{d} t+\Psi(u)-\Psi(v) \\
& \quad \geq-\frac{1}{2}\left\|v_{0}-u(0)\right\|^{2} .
\end{aligned}
$$

Since $\left\|B_{2}(v(t), v(t))\right\|_{\mathbf{V}^{*}} \leq K\|v(t)\|_{\mathbf{V}}^{3 / 2}\|v(t)\|^{1 / 2}$ (cf. (165)) and $v \in L^{\infty}\left(0, T ; \mathbf{L}^{2}\right), B_{2}(v, v) \in L^{4 / 3}\left(0, T ; \mathbf{V}^{*}\right)$. Therefore, by density of the set $\left\{u=\sum_{j=1}^{M} k_{j}(t) u_{j}, k_{j}(t) \in C^{1}[0, T], M=\right.$
$1,2, \ldots\}$ in $\left\{L^{4}(0, T ; \mathbf{V}): u^{\prime} \in L^{2}\left(0, T ; \mathbf{V}^{*}\right)\right\},(195)$ is valid for all $u \in\left\{L^{4}(0, T ; \mathbf{V}): u^{\prime} \in L^{2}\left(0, T ; \mathbf{V}^{*}\right)\right\}$.

Thus, the first formula of (43) is valid.

Putting $\varphi(t)=\sum_{j=1}^{M} k_{j}(t) \varphi_{j}, \quad$ where $k_{j}(t) \in C^{1}[0, T]$, $k_{j}(T)=0$, multiply the second equation of $(141)$ by $k_{j}(t)$ and add for $j=1, \ldots, M$. Thus, we have

$$
\begin{aligned}
& \left\langle\theta_{m}(t), \varphi(t)\right\rangle-\int_{0}^{t}\left\langle\theta_{m}, \frac{\partial \varphi}{\partial t}\right\rangle \mathrm{d} s+\int_{0}^{t} \kappa\left(\theta_{m} \nabla \theta_{m}, \nabla \varphi\right) \mathrm{d} s \\
& \quad+\int_{0}^{t}\left(\beta(x) \theta_{m}, \varphi\right)_{\Gamma_{R}} \mathrm{~d} s-\int_{0}^{t}\left\langle v_{m} \theta_{m}, \nabla \varphi\right\rangle \mathrm{d} s \\
& =\left\langle\theta_{m}(0), \varphi(0)\right\rangle+\int_{0}^{t}\left\langle g_{2}, \varphi\right\rangle \mathrm{d} s, \quad \forall t \in[0, T] .
\end{aligned}
$$

By Corollary Appendix B.2 of [1], we have

$$
\begin{aligned}
& \int_{0}^{t}\left(\kappa\left(\theta_{m}\right) \nabla \theta_{m}, \nabla \varphi\right) \mathrm{d} s \longrightarrow \int_{0}^{t}(\kappa(\theta) \nabla \theta, \nabla \varphi) \mathrm{d} s, \quad \text { as } \\
& \quad m \longrightarrow \infty, \varepsilon \longrightarrow 0 .
\end{aligned}
$$

Since $W^{9 / 10,2}(\Omega) \subset \mathbf{L}^{4}(\Omega)$, we have

$$
\begin{aligned}
& v_{m} \longrightarrow v \operatorname{in} L^{2}\left(0, T ; \mathbf{L}^{4}(\Omega)\right), \\
& \theta_{m} \longrightarrow \theta \operatorname{in} L^{2}\left(0, T ; L^{4}(\Omega)\right) .
\end{aligned}
$$

By (198), we have

$$
\begin{aligned}
\int_{0}^{t}\left|\left\langle v_{m} \theta_{m}, \nabla \varphi\right\rangle-\langle v \theta, \nabla \varphi\rangle\right| \mathrm{d} s & \leq \int_{0}^{t}\left|\left\langle\left(v_{m}-v\right) \theta_{m}, \nabla \varphi\right\rangle\right| \mathrm{d} s+\int_{0}^{t}\left|\left\langle v\left(\theta_{m}-\theta\right), \nabla \varphi\right\rangle\right| \mathrm{d} s \\
& \leq\left(\left\|v_{m}-v\right\|_{L^{2}\left(0, T ; \mathbf{L}^{4}\right)}\left\|\theta_{m}\right\|_{L^{2}\left(0, T ; L^{4}\right)}+\|v\|_{L^{2}\left(0, T ; \mathbf{L}^{4}\right)}\left\|\theta_{m}-\theta\right\|_{L^{2}\left(0, T ; L^{4}\right)}\right)\|\nabla \varphi\|_{L^{\infty}\left(0, T ; \mathbf{L}^{2}\right)} \longrightarrow 0
\end{aligned}
$$

which implies

$$
\int_{0}^{t}\left\langle v_{m} \theta_{m}, \nabla \varphi\right\rangle \mathrm{d} s \longrightarrow \int_{0}^{t}\langle v \theta, \nabla \varphi\rangle \mathrm{d} s, \quad \text { as } m \longrightarrow \infty, \varepsilon \longrightarrow 0 .
$$

Therefore, taking into account (197) and (200), from (196), we have

$$
\begin{aligned}
& -\int_{0}^{T}\left\langle\theta, \frac{\partial \varphi}{\partial t}\right\rangle \mathrm{d} t+\int_{0}^{T}(\kappa(\theta) \nabla \theta, \nabla \varphi) \mathrm{d} t+\int_{0}^{T}(\beta(x) \theta, \varphi)_{\Gamma_{R}} \mathrm{~d} t \\
& -\int_{0}^{T}\langle v \theta, \nabla \varphi\rangle \mathrm{d} t \\
& \quad=\langle\theta(0), \varphi(0)\rangle+\int_{0}^{t}\left\langle g_{2}, \varphi\right\rangle \mathrm{d} s .
\end{aligned}
$$

Since the set $\left\{\varphi(t)=\sum_{j=1}^{M} k_{j}(t) \varphi_{j}: k_{j}(t) \in C^{1}[0\right.$, $\left.T], k_{j}(T)=0, M=1,2, \ldots\right\} \quad$ is dense in $\left\{\varphi \in C^{1}\left([0, T] ; W_{\Gamma_{D}}^{1,2}\right): \varphi(T)=0\right\}$, from (201), we have the second equation of (43).

\section{Data Availability}

No data were used to support this study.

\section{Conflicts of Interest}

The author declares that there are no conflicts of interest.

\section{Acknowledgments}

The author was supported by the Scientific and Technical Development Plan Fund SCDP-5. 


\section{References}

[1] T. Kim and D. Cao, "A non-steady system with friction boundary conditions for flow of heat-conducting incompressible viscous fluids," Journal of Mathematical Analysis and Applications, vol. 484, no. 1, Article ID 123676, 2020.

[2] H. Inoue, "On heat convection equations with dissipative terms in time dependent domains," Nonlinear Analysis: Theory, Methods \& Applications, vol. 30, no. 7, pp. 4441-4448, 1997.

[3] H. Inoue, "Strong solutions of two dimensional heat convection equations with dissipating terms," Tokyo Journal of Mathematics, vol. 22, no. 2, pp. 445-472, 1999.

[4] H. Inoue, "Global and periodic solutions of heat convection equations with internal friction terms," Nonlinear Analysis: Theory, Methods \& Applications, vol. 47, no. 4, pp. 2881-2886, 2001.

[5] G. Łukaszewicz and P. Krzyżanowski, "On the heat convection equations with dissipation term in regions with moving boundaries," Mathematical Methods in the Applied Sciences, vol. 20, no. 4, pp. 347-368, 1997.

[6] H. Morimoto, "On non-stationary Boussinesq equations," Proceedings of the Japan Academy, Series A, Mathematical Sciences, vol. 67, no. 5, pp. 159-161, 1991.

[7] H. Morimoto, "Non-stationary Boussinesq equations," J. Fac. Sci. Univ. Tokyo Sect. IA Math.vol. 39, pp. 61-75, 1992.

[8] H. Morimoto, "Heat convection equation with nonhomogeneous boundary condition," Funkcialaj Ekvacioj, vol. 53, no. 2, pp. 213-229, 2010.

[9] H. Morimoto, "Survey on time periodic problem for fluid flow under inhomogeneous boundary condition," Discrete \& Continuous Dynamical Systems, vol. 5, no. 3, pp. 631-639, 2012.

[10] H. Inoue and M. Ôtani, "Strong solutions of initial boundary value problems for heat convection equations in noncylindrical domains," Nonlinear Analysis: Theory, Methods \& Applications, vol. 24, no. 7, pp. 1061-1080, 1995.

[11] H. Inoue and M. Ôtani, "Periodic problems for heat convection equations in noncylindrical domains," Funkcialaj Ekvacioj, vol. 40, pp. 19-39, 1997.

[12] K. Oeda, "On the initial value problem for the heat convection equation of Boussinesq approximation in a time-dependent domain," Proceedings of the Japan Academy, Series A, Mathematical Sciences, vol. 64, pp. 143-146, 1988.

[13] K. Oeda, "Remarks on the periodic solution of the heat convection equation in a perturbed annulus domain," Proceedings of the Japan Academy, Series A, Mathematical Sciences, vol. 73, pp. 21-25, 1997.

[14] K. Oeda, "Periodic solutions of the heat convection equations in exterior domains," Proceedings of the Japan Academy, Series A, Mathematical Sciences, vol. 73, pp. 49-54, 1997.

[15] K. Oeda and N. Matsuda, "Initial value problems for the heat convection equations in exterior domains," Tokyo Journal of Mathematics, vol. 21, no. 2, pp. 359-375, 1998.

[16] Z. Skalák and P. Kučera, "An existence theorem for the Boussinesq equations with non-Dirichlet boundary conditions," Applications of Mathematics, vol. 45, no. 2, pp. 81-98, 2000.

[17] P. Kacprzyk, "Long-time existence of solutions to the NavierStokes equations with inflow-outflow and heat convection," Mathematical Methods in the Applied Sciences, vol. 35, no. 9, pp. 1000-1013, 2012.

[18] T. Kim and D. Cao, "Existence of solutions to the heat convection equations in a time-dependent domain with mixed boundary conditions," Journal of Mathematical Sciences University of Tokyo, vol. 22, pp. 531-568, 2015.

[19] T. Kim and D. Cao, "Some properties on the surfaces of vector fields and its application to the Stokes and Navier-Stokes problems with mixed boundary conditions," Nonlinear Analysis: Theory, Methods \& Applications, vol. 113, pp. 94114, 2015.

[20] T. Kim and D. Cao, "Non-stationary Navier-Stokes equations with mixed boundary conditions," Journal of Mathematical Sciences University of Tokyo, vol. 24, pp. 159-194, 2017.

[21] T. Kim and F. Huang, "The non-steady Navier-Stokes systems with mixed boundary conditions including friction conditions," Methods and Applications of Analysis, vol. 25, no. 1, pp. 13-50, 2018.

[22] J. Naumann and J. Wolf, "Existence of weak solutions to the equations of natural convection with dissipative heating," Advances in Mathematical Fluid Mechanics, pp. 367-384, Springer, Berlin, Germany, 2010.

[23] M. Beneš, "Mixed initial-boundary value problem for the three-dimentional Navier-Stokes equations in polyhedral domains," Discrete \& Continuous Dynamical Systems, vol. 2, pp. 135-144, 2011.

[24] T. Kim and D. Cao, "The steady Navier-Stokes and Stokes systems with mixed boundary conditions including one-sided leaks and pressure," Methods and Applications of Analysis, vol. 23, no. 4, pp. 329-364, 2016.

[25] H. Gajewski, K. Groger, and K. Zacharias, Nichtlinear Operatorgleichungen und Operatordifferentialgleichungen, Academie-Verlag, Berlin, Germany, 1974.

[26] J. Naumann, "On evolution inequalities of Navier-Stokes type in three dimensions Ann," Annali di Matematica Pura ed Applicata, vol. 124, no. 4, pp. 107-125, 1980.

[27] V. Barbu, Nonlinear Differential Equations of Monotone Types in Banach Spaces, Springer, Berlin, Germany, 2010.

[28] P. Grisvard, Elliptic Problems in Nonsmooth Domains, Pitman Advanced Publishing Program Boston London Melbourne, SIAM Philadelphia, Philadelphia, PA, USA, 1985.

[29] J. Simon, "Compact sets in the space $L^{p}(0, T ; B)$." Annali di Matematica Pura ed Applicata, vol. 146, no. 4, pp. 65-96, 1987. 